\title{
The influence of solar wind on extratropical cyclones - Part 2: A link mediated by auroral atmospheric gravity waves?
}

\author{
P. Prikryl ${ }^{1}$, D. B. Muldrew ${ }^{1}$, and G. J. Sofko ${ }^{2}$ \\ ${ }^{1}$ Communications Research Centre Canada, Ottawa, Canada \\ ${ }^{2}$ University of Saskatchewan, Saskatoon, Canada
}

Received: 24 July 2007 - Revised: 19 August 2008 - Accepted: 22 October 2008 - Published: 6 January 2009

\begin{abstract}
Cases of mesoscale cloud bands in extratropical cyclones are observed a few hours after atmospheric gravity waves (AGWs) are launched from the auroral ionosphere. It is suggested that the solar-wind-generated auroral AGWs contribute to processes that release instabilities and initiate slantwise convection thus leading to cloud bands and growth of extratropical cyclones. Also, if the AGWs are ducted to low latitudes, they could influence the development of tropical cyclones. The gravity-wave-induced vertical lift may modulate the slantwise convection by releasing the moist symmetric instability at near-threshold conditions in the warm frontal zone of extratropical cyclones. Latent heat release associated with the mesoscale slantwise convection has been linked to explosive cyclogenesis and severe weather. The circumstantial and statistical evidence of the solar wind influence on extratropical cyclones is further supported by a statistical analysis of high-level clouds $(<440 \mathrm{mb})$ extracted from the International Satellite Cloud Climatology Project (ISCCP) D1 dataset. A statistically significant response of the high-level cloud area index (HCAI) to fast solar wind from coronal holes is found in mid-tohigh latitudes during autumn-winter and in low latitudes during spring-summer. In the extratropics, this response of the HCAI to solar wind forcing is consistent with the effect on tropospheric vorticity found by Wilcox et al. (1974) and verified by Prikryl et al. (2009). In the tropics, the observed HCAI response, namely a decrease in HCAI at the arrival of solar wind stream followed by an increase a few days later, is similar to that in the northern and southern mid-to-high latitudes. The amplitude of the response nearly doubles for stream interfaces associated with the interplanetary magnetic field $B_{Z}$ component shifting southward. When the IMF $B_{Z}$ after the stream interface shifts northward, the autumn-winter
\end{abstract}

Correspondence to: P. Prikryl (paul.prikryl@crc.ca) effect weakens or shifts to lower (mid) latitudes and no statistically significant response is found at low latitudes in springsummer. The observed effect persists through years of low and high volcanic aerosol loading. The similarity of the response in mid-to-high and low latitudes, the lack of dependence upon aerosol loading, and the enhanced amplitude of the effect when IMF $B_{Z}$ component shifts southward, favor the proposed AGW link over the atmospheric electric circuit (AEC) mechanism (Tinsley et al., 1994). The latter requires the presence of stratospheric aerosols for a significant effect and should produce negative and positive cloud anomalies in mid-to-high and low latitudes, respectively. However, if the requirement of aerosols for the AEC mechanism can be relaxed, the AGW and AEC mechanisms should work in synergy at least in mid-to-high latitudes.

Keywords. Meteorology and atmospheric dynamics (Convective processes; Mesoscale meteorology; Waves and tides)

\section{Introduction}

A correlation has been found between the solar wind and tropospheric circulation on the scale of days (Wilcox et al., 1973, 1974, 1976). That correlation involves the relationship between the passage of the Earth through the solar wind magnetic sector boundary or heliospheric current sheet (HCS) and a decrease in the upper-level tropospheric vorticity, as found by Wilcox et al. The vorticity area index (VAI) that was introduced by Roberts and Olson (1973) was used in superposed epoch analysis that showed the "Wilcox effect", namely a decrease in VAI one day after the HCS passage. These results were shown to be statistically significant by Hines and Halevy (1977) who concluded that an unknown "operative solar mechanism provides its signal by means of a modulation of meteorological variations", very likely a

Published by Copernicus Publications on behalf of the European Geosciences Union. 
phase modulation. However, an acceptable mechanism has not been identified.

The possibility of galactic cosmic ray (GCR) influence on clouds has been studied but reassessment of the correlations found by Svensmark and Friis-Christensen (1997) and Marsh and Svensmark $(2000,2004)$ raised doubts about the longterm stability of the correlations over interannual timescales (Sun and Bradley, 2002). Todd and Knivelton (2001) focused on day-to-day effects of Forbush decreases (FD) in GCRs and found small but significant decline in cloud cover following the FD events. Reviews of observations, theories and possible mechanisms have been published by Carslaw et al. (2002) and Harrison and Carslaw (2003) but the GCR mechanism remains controversial.

Tinsley et al. (1994) suggested a mechanism involving global-circuit atmospheric electric current (AEC) through clouds and changes in relativistic electron precipitation linking the effects of charge accumulation to changes in ice nucleation. This idea has been vigorously pursued in both observational and modelling work (Kirkland et al., 1996; Kniveton and Tinsley, 2004; Roldugin and Tinsley, 2004; Tinsley, 2000; Tinsley et al., 2001, 2006, 2007; Burns et al., 2007; Tinsley and Zhou, 2006; Zhou and Tinsley, 2007). As for the relationship of the AEC mechanism to the Wilcox effect, the VAI minimum following the HCS transit is hypothesized to be caused by a reduction in the scavenging of contact-ice nuclei by supercooled droplets, in the presence of stratospheric aerosols (Tinsley et al., 1994). This would lead to a reduction in the rate of glaciation of the winter storm clouds, and reduction of precipitation efficiency and latent heat production and its redistribution (Tinsley and Deen, 1991).

In the companion paper, Prikryl et al. (2009) use the ECMWF ERA-40 re-analysis dataset for a period from 1963 to 2002 to verify the Wilcox effect for the northern as well as the Southern Hemisphere winters. It is shown that the vorticity area index (VAI) at 300 and $500 \mathrm{mb}$ is modulated by highspeed solar wind streams flowing from coronal holes. Also, a strong tendency for significant extratropical storms to occur within a few days after the high-speed solar wind arrival is found. This is shown for lists of major extratropical storm events as well as significant sea level pressure deepenings of extratropical storms for a large database of storm tracks. In this paper (Sect. 7), these results are corroborated by a statistical analysis of high-level clouds $(<440 \mathrm{mb})$ extracted from the International Satellite Cloud Climatology Project (ISCCP) D1 dataset.

These new observations, the verification of the link between the solar wind and the upper troposphere, and the persistence of the effect through years of high and low volcanic aerosol loading, underline the need to find an acceptable mechanism. One tenet of the AEC mechanism is now in question, namely the extent of the role of aerosols. However, the nature of the mechanism to explain the observed sun-weather link and its characteristics are not yet fully understood (Carslaw et al., 2002).

In this paper (Sects. 5 and 6) it is proposed that auroral atmospheric gravity waves (AGWs) may release symmetric instabilities leading to slantwise convection and latent heat release, which is the energy source for storminess. While definitive proof for such a mechanism linking solar wind and tropospheric processes remains to be demonstrated, we believe that proposing a concept and raising awareness of such a possibility will eventually lead to a better understanding of the observed link. It is noted that, contrary to the AEC model, the AGW mechanism does not require the presence of aerosols in the atmosphere. Also, the AGW mechanism would produce similar effects in weather systems in the midto-high and low latitude regions.

Two case studies (Sects. 4 and 5) are presented to suggest that auroral atmospheric gravity waves (AGWs) may influence the formation of mesoscale bands and development of extratropical cyclones. Readers may initially proceed to Case 2 which should suffice to convey the general idea that solar wind coupling to the ionosphere can produce auroral AGWs, which in turn couple to the troposphere. The circumstantial evidence for such a link is then supported by statistical results (Sect. 7) of the superposed epoch (SPE) analysis of high-level cloud area index (HCAI) derived from the ISCCP-D1 data. Time series of HCAI are keyed by the arrival time of corotating interaction regions at the leading edge of high-speed solar wind streams.

Invoking auroral atmospheric gravity waves as a link between the solar wind and troposphere necessitates a brief introduction to: (a) solar wind coupling to the magnetosphereionosphere-thermosphere (MIT) system; (b) generation and propagation of gravity waves; (c) auroral AGW coupling to mesoscale processes in the troposphere.

\subsection{Auroral atmospheric gravity waves and traveling ionospheric disturbances}

High-speed solar wind plasma streams from coronal holes are the major carriers of solar wind energy flow towards the Earth. As the fast solar wind overtakes the slower solar wind plasma, the interaction between the two regions generates large-amplitude magneto-hydrodynamic (MHD) waves and shocks. The fast solar wind is permeated with plasma fluctuations, particularly in the interplanetary magnetic field (IMF) and ion velocity components, which are due to Alfvén waves (Belcher and Davis, 1971) and are thought to be the remnants of waves generated near the Sun. The solar wind energy that couples to the magnetosphere drives ionospheric convection and auroral electrojets. The bulk of highly variable solar wind energy is channelled to the auroral ovals and deposited in the upper atmosphere, much of it in the form of Joule heating. On time scales from tens of minutes to a few hours, auroral electrojets and ionospheric flow bursts generate atmospheric gravity (buoyancy) waves by Joule heating 
or Lorentz forcing (Chimonas and Hines, 1970). Based on later studies (Hunsucker, 1977; Brekke, 1979) the Lorenz forcing is dominant and is assumed by Luhmann (1980) in modeling of the low-frequency pressure and velocity perturbations near the auroral electrojet. The atmospheric gravity waves interact with neutral winds and deposit their momentum in the atmosphere. A causal relationship between atmospheric gravity waves (AGWs) and traveling ionospheric disturbances (TIDs) has been well established (Hocke and Schlegel, 1996).

Theoretical understanding of AGWs and their role in the ionosphere was developed by Hines (1960). The gravity wave dispersion relation allows both upward group (downward phase) and downward group (upward phase) propagation. Based on a spectral model in terms of spherical harmonics Mayr et al. (1984a, b) described gravity wave response in the atmosphere and showed that propagating waves originating in the thermosphere can excite a spectrum of AGWs in the lower atmosphere, though with much smaller amplitudes. For medium-scale AGWs generated by auroral sources, Francis (1974) distinguished between direct and earth-reflected AGWs. He pointed out that the latter appear in the ionosphere as monochromatic wave packets while the former induce isolated TIDs.

Identification of sources of medium-scale TIDs has remained difficult. Both the auroral activity (e.g. Hunsucker, 1982; Lewis et al., 1996) and tropospheric weather disturbances (e.g. Bertin et al., 1975; Waldock and Jones, 1987; Oliver et al., 1997) can generate medium-scale AGWs, particularly for TIDs that are observed at middle latitudes. At high latitudes, TIDs that were attributed to earth-reflected AGWs originating near the ionospheric convection reversal boundary were observed with HF radar by Samson et al. (1989, 1990). Such radars are now a part of SuperDARN (Super Dual Auroral Radar Network) (Greenwald et al., 1995; Chisham et al., 2007). TIDs modulate the radar ground-scatter (Bristow and Greenwald, 1996) while at the same time, their sources may be observed in the high-latitude ionosphere, particularly in the dayside auroral oval (e.g. Bristow et al., 1994; Prikryl et al., 2005). These observations are consistent with modeling studies showing that each pulse of the thermospheric electric field launches one cycle of a gravity wave (Millward et al., 1993a, b; Millward, 1994).

\subsection{Atmospheric gravity waves in the lower atmosphere}

In both the middle and lower atmosphere, dominant sources of AGWs are identified in the troposphere and include topography, convection, wind shear and geostrophic adjustment (Fritts and Alexander, 2003). Gravity waves can modulate cloud formation by initiating and organizing convective activity (Uccellini, 1975; Lindzen and Tung, 1976). It has been recognized that AGWs can significantly and often unpredictably impact the development of mesoscale weather systems. Operational forecasting and detection of AGWs has become an important component of the weather forecasting process (Koch and O'Handley, 1997).

Measurements of horizontal winds in the mesosphere and lower thermosphere showed that at least 65 percent of the vertical energy flux of long period gravity waves is upgoing (Vincent, 1984). Fritts and Alexander (2003), after reviewing observational approaches to determine the fractions of the AGW spectrum that propagate upward and downward, suggested that the fraction of upward propagating AGW energy is much greater than $1 / 2$. As far as we know, little attention has been given to the possibility that down-going auroral gravity wave energy affects the lower atmosphere. The reason is apparent: in a stationary atmosphere in hydrostatic equilibrium, the gravity wave amplitude decreases exponentially with decreasing height. At cloud level the amplitude in velocity perturbation is three orders of magnitude smaller than the amplitude of the waves launched in the Eregion (Hines, 1965). Luhmann (1980) theoretically investigated the possibility that auroral-electrojet-generated gravity waves affect the development of air turbulence in the middle atmosphere. Using a model of an isothermal, windless atmosphere perturbed by gravity waves generated by an intense, exceptionally thin electrojet, significant near-field pressure and velocity perturbations were computed. Because the auroral conditions modeled by Luhmann were rather extreme, Cole (1984) concluded that gravity waves launched by the auroral electrojet generally do not cause significant perturbation of the wind fields in the middle atmosphere. However, at altitudes $110-120 \mathrm{~km}$, upward and downward vertical winds in excess of $30 \mathrm{~m} / \mathrm{s}$ have been observed at high latitudes during moderately-disturbed geomagnetic conditions (Oyama et al., 2008). Rees et al. (1984) identified sources at high latitudes of strong vertical winds of more than $100 \mathrm{~m} / \mathrm{s}$, resulting from local geomagnetic energy input and subsequent generation of thermospheric gravity waves. Previous observations (e.g. Hernandez, 1982) of periodic oscillations with amplitudes up to $50 \mathrm{~m} / \mathrm{s}$ and period of $40 \mathrm{~min}$ in the mid-latitude thermosphere were attributed to the passage of gravity waves from an auroral source. Such vertical motions scale down to a few $\mathrm{cm} / \mathrm{s}$ or more at the tropospheric level, which is comparable to mean vertical motions in the troposphere (e.g. Fukao et al., 1991; Nastrom and VanZandt, 1994) but less than typical instantaneous vertical motions of up to a few tens of $\mathrm{cm} / \mathrm{s}$ associated with tropospheric gravity waves (Nastrom et al., 1990).

Because there is an exchange of energy between the waves and the wind, under certain circumstances the waves can be amplified (McKenzie, 1972), with the waves drawing energy from the wind. Although such non-linear amplification of gravity waves is not well understood, the possibility that ionospherically forced gravity waves influence the lower atmosphere cannot be discounted. Radar observations of earthreflected gravity waves (Samson et al., 1989) imply that auroral gravity waves are not negligible at ground level. It is 
conceivable that they may release instabilities in the troposphere leading to convection and production of cloud (precipitation) bands and thus play a role in development of extratropical cyclones.

Single- and multiple-banded clouds and precipitation can be a manifestation of various tropospheric processes, including frontogenesis, boundary layer instabilities, ducted gravity waves, the Kelvin-Helmhotz instability, and moist slantwise convection due to the release of the moist symmetric instability (MSI) (Schultz and Schumacher, 1999, and references therein). The "ingredients-based methodology" for forecasting deep, moist convection requires three ingredients: instability, moisture and lift (e.g. Doswell et al., 1996). One type of MSI, which is the conditional symmetric instability (CSI), has commonly been invoked to explain cloud/precipitation bands (Schultz and Schumacher, 1999). However, as discussed in detail by these authors, the existence of the instability alone is not sufficient to initiate convection. The required lift has to be provided by some other process, e.g. frontogenesis. Infinitesimal perturbations, as opposed to finite-amplitude forcing, have also been considered for the release of CSI. It is believed that the majority of CSI-related phenomena observed are related to finiteamplitude forcing (Schultz and Schumacher, 1999). Here we present observations that suggest that the lift can be provided by auroral AGWs. The estimated wave vertical velocity amplitudes of a few $\mathrm{cm} / \mathrm{s}$ or more are comparable to mean vertical motions in the troposphere and may contribute to updrafts involved in slantwise convection initiation. Thus the auroral AGWs may add enough vertical motion to release the instability and initiate a slantwise convection, as discussed in Sect. 6.

From a historical perspective, early ideas on extratropical circulation and cyclones that were developed by the Bergen school founded by Vilhelm Bjerknes (Thorpe, 2002) originated from attempts to draw an analogy between hydrodynamic and electromagnetic phenomena (Bjerknes, 1898). One source of auroral AGWs is the coupling between the solar wind MHD waves (Alfvén, 1942) and the magnetosphereionosphere system that, in turn, is coupled to the neutral atmosphere. The coupling thus provides a link between the initial electromagnetic and the final hydrodynamic phenomena.

\section{Instruments, techniques and data}

SuperDARN is an international network of HF radars primarily designed to monitor ionospheric convection (Greenwald et al., 1995; Chisham et al., 2007). The radars transmit at frequencies $8-20 \mathrm{MHz}$ along 16 successive beams, each of which is gated into 75 range bins. The bins are $45 \mathrm{~km}$ long in standard operations, and the dwell time for each beam is $7 \mathrm{~s}$. A full 16-beam scan with successive beams separated by $3.25^{\circ}$ covers $52^{\circ}$ in azimuth every $2 \mathrm{~min}$. Several parameters including the line-of-sight Doppler velocity, spectral width and backscatter power from ionospheric plasma irregularities are routinely measured. The 16 beams forming each radar field-of-view are numbered clockwise. In the standard mode the two line-of-sight velocities measured at the intersection of the beams from two radars are combined to provide the convection velocity component perpendicular to the magnetic field. Global ionospheric convection and potential maps are derived by fitting the available velocity data to an expansion of the potential in terms of spherical harmonic functions (Ruohoniemi and Baker, 1998). Where radar echoes are absent, data from a statistical convection model are included to constrain the solution. The AGWs are observed as TIDs that are seen as moving bands in the ground scatter power. This power is modulated by the focusing and defocusing of the HF signals reflected from the lower F-region undulations caused by the passage of the gravity waves (Samson et al., 1989).

Advanced Communications Technology Satellite (ACTS) beacon signals at 20.2 and $27.5 \mathrm{GHz}$ have been received at the Communications Research Centre Canada, Ottawa $\left(45.61^{\circ} \mathrm{N}, 75.62^{\circ} \mathrm{W}\right)$, for radio propagation studies (Amaya and Rogers, 2000, 2002) over a satellite link at an elevation of $32.2^{\circ}$ and an azimuth of $212.4^{\circ}$ (clock-wise from geographic north). Simultaneously, radiometric sky brightness temperatures (RSBT) at 12, 20 and $30 \mathrm{GHz}$ have been monitored. The ACTS beacon signal and radiometer data sampled at a $1-\mathrm{Hz}$ rate have been used to assess the signal attenuation due to cloud or precipitation, including seasonal and inter-annual characteristics of signal fading (Amaya and Rogers, 2002). Quasi-periodic signal fades and associated RSBT enhancements can indicate passing cloud or precipitation bands.

Imagers on the Geostationary Operational Environmental Satellites (GOES) and Meteosat satellites are multichannel instruments that sense radiant energy and reflected solar energy from the Earth's surface and atmosphere. The satellite infrared (IR) images of clouds are used to identify mesoscale cloud bands (horizontal dimensions of about $100 \mathrm{~km}$ in width and several hundred $\mathrm{km}$ in length). The images were obtained from the Meteorological Service of Canada (MSC), National Oceanic and Atmospheric Administration (NOAA) and the European Meteorological Satellite Organization (EUMETSAT).

The ISCCP-D1 dataset (Rossow and Schiffer, 1999), that provides global climatology of cloud coverage and numerous cloud parameters derived from meteorological satellite imagery, is currently available for the period from July 1983 through June 2006. Cloud cover of various types (Hahn et al., 2001) based on the fraction of cloudy pixels is determined on a grid of $280-\mathrm{km}$ by $280-\mathrm{km}$ cells.

Ground-based magnetometer data from the Danish Meteorological Institute (DMI) Greenland chain (Stauning et al., 1994), the MACCS array (Hughes and Engebretson, 1997), the Canadian GeoSpace Monitoring (CGSM) network, formerly CANOPUS (Rostoker et al., 1995), and the magnetic 
field measurements at geosynchronous orbit from the GOES10 spacecraft, are used to identify TID sources.

The National Space Science Data Center (NSSDC) OMNIWeb http://omniweb.gsfc.nasa.gov/ provides the solar wind plasma and interplanetary magnetic field (IMF) database that is assembled from observations by many spacecraft - primarily the ISEE and IMP series, Wind, and ACE starting in 1963. Some plasma data gaps are filled with data from the Proton Monitor on the Solar and Heliospheric Observatory (SOHO).

Intensity measurements since 1939 of the green coronal emission line (Fe XIV, $530.3 \mathrm{~nm}$ ) were obtained by several ground-based coronagraphs and have been merged into a homogeneous coronal dataset based on the Lomnický Štít photometric scale since 1965 (Rybanský et al., 2001, 2005). The coronal intensities measured at a height of 40 arcseconds above the solar limb are expressed in absolute coronal units (ACU) (ftp://ftp.ngdc.noaa.gov/stp/solar_data). One ACU represents the intensity of the continuous spectrum from the center of the solar disk with a width of $1 \AA$ at the same wavelength as the observational spectral line $\left(1 \mathrm{ACU}=3.89 \mathrm{~W} \mathrm{~m}^{-2} \mathrm{sr}^{-1}\right.$ at $\left.530.3 \mathrm{~nm}\right)$. Synoptic charts of the green corona intensity at the solar central meridian are constructed from these data (Prikryl et al., 2009). From November 1991 to December 2001, the soft X-ray solar images taken from Yohkoh spacecraft (http://www.lmsal.com/ sxt/), and starting from 1996 the SOHO EUV images (http: //umbra.nascom.nasa.gov/eit/), are also used to identify coronal holes.

\section{Ray tracing of gravity waves}

For a spherically stratified atmosphere with no horizontal gradients a simplified ray tracing procedure based on Snell's law is applicable. The dispersion relation between the angular wave frequency $\omega$ and wave vector $\boldsymbol{k}$ given by Hines (1960) is used:

$\left(\omega^{2}-\omega_{a}^{2}\right) \omega^{2} / C^{2}-\omega^{2}\left(k_{x}^{2}+k_{z}^{2}\right)+\omega_{b}^{2} k_{x}^{2}=0$,

where $\omega_{a}=\gamma g / 2 C$ is the acoustic cutoff frequency, $\gamma, C, g$ are the ratio of specific heats, speed of sound, and acceleration due to gravity, respectively, and $k_{x}$ and $k_{z}$ are the horizontal and vertical components of the wave vector $\boldsymbol{k}$. For an atmosphere whose scale height varies with altitude, the Brunt-Väisälä (buoyancy) frequency $\omega_{b}$ is defined as

$\omega_{b}^{2}=(\gamma-1) g^{2} / C^{2}+\left(g / C^{2}\right)\left(d C^{2} / d z\right)$

The atmosphere is divided into horizontal slabs of constant temperature and density that are obtained from the MSIS 90 model (Hedin, 1991). Viscosity, thermal conductivity and, initially, neutral winds are neglected. Subsequently, the effect of neutral winds is briefly considered.

The ray tracing is initiated at an electrojet altitude of $110 \mathrm{~km}$ starting with initial values $\alpha_{0}$ of the $\boldsymbol{k}$ directions $\alpha$, where $\alpha$ is taken to be positive upward from the southward horizontal direction in the Northern Hemisphere. The group velocity $\mathbf{V}_{g r} \equiv\left(\partial \omega / \partial k_{x}, 0, \partial \omega / \partial k_{z}\right)$ or path of energy flow of a 40-min gravity wave is obtained from Eq. (1) numerically. For $\alpha_{0}<0$ (downward phase velocity), the group direction is upward and the gravity wave energy escapes upwards through the F-region of the ionosphere within a relatively short horizontal distance of less than $1000 \mathrm{~km}$. For $\alpha_{0}>0$, the initial group direction is downward, so the gravity waves would be expected to reach the surface of the earth unless they are reflected.

For a windless atmosphere and a range of initial values $\alpha_{0}$ stepped by $0.2^{\circ}$, Fig. 1a-d shows rays that are reflected by the temperature gradient in the upper mesosphere, as well as downward rays $\left(\alpha_{0}>83.6^{\circ}\right)$ reaching the troposphere after more than $2 \mathrm{~h}$. The MSIS90 atmospheric temperature profile for 17 November 2001 (Case 2) is shown. Panels (a-d) have the same rays, colour-coded to show the value of 4 different parameters: time of travel $t$, horizontal and vertical wavelengths $\lambda_{x}$ and $\lambda_{z}$, respectively, and horizontal phase velocity $V_{x}^{p h}$.

The vertical profile of the atmospheric scale height that determines the ray path varies diurnally, seasonally and with the solar cycle. In addition, short-term variations on the scale of days due to solar-wind-induced geomagnetic activity also affect the scale height, particularly near the mesopause and above (Lindblad, 1978, 2003; Prikryl, 1979). Such dependencies are expected to affect the reflection/penetration of auroral AGWs near the mesopause. In regions of steep atmospheric temperature (and hence density scale height) gradients, particularly in the upper mesosphere and troposphere, the vertical wavelength $\lambda_{z}$ increases and may become imaginary, in which case the wave is reflected (e.g. Prikryl et al., 2005, their Fig. 3).

The validity of ray tracing near the reflection height, where $1 / k_{z}$ can be greater than the neutral density scale height, is discussed by Prikryl et al. (2005). Although the ray paths may not be valid near the reflection height, the computed rays are valid a few tens of kilometers above that height. For $\alpha_{0}<83.5^{\circ}$, most of the downward propagating energy is reflected in the upper mesosphere. However, part of the wave energy would tunnel below the reflection point in the form of an evanescent wave. Once the refractive index becomes real at lower altitudes the gravity wave will propagate again. Thus it is likely that the actual downward AGW flux is not limited to the downward rays below the mesosphere based on geometric optics as shown in Fig. 1. Also, there is an amplification of the wave due to reflection since $\partial \omega / \partial k_{z} \rightarrow 0$ but full wave theory is required to assess it.

For the downward group paths, the horizontal wavelength $\lambda_{x}$ and phase velocity $V_{x}^{p h}$ decrease with increasing $\alpha_{0}$. The phase velocity of the downward transmitted AGWs becomes comparable to the wind velocity, in which case the Dopplershifted frequency has to be used in Eq. (1). This may lead to 

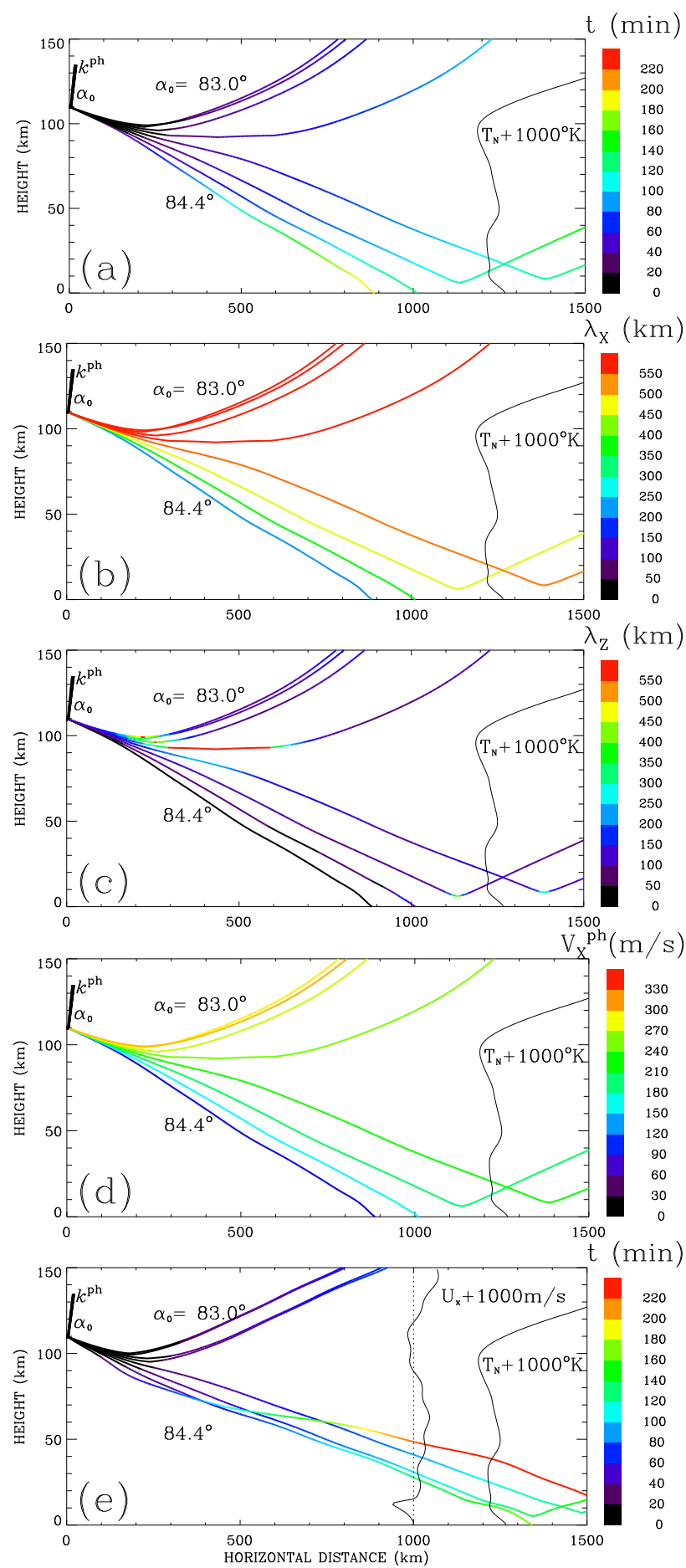

Fig. 1. Ray paths for 40-min gravity waves (a-d) in a stationary MSIS90 model atmosphere and (e) for a simulated wind profile, $U_{x}(z)$. The rays are colour-coded to show the values of different parameters $\left(t, \lambda_{x}, \lambda_{z}, V_{x}^{p h}\right)$. The neutral temperature $T_{N}(z)$ and wind $U_{x}(z)$ profiles are shown using the abscissa with origin shifted and scale units in ${ }^{\circ} \mathrm{K}$ and $\mathrm{m} / \mathrm{s}$, respectively. ducting or reflection of gravity waves (Cowling et al., 1971; Yeh and Lin, 1974; Chimonas and Hines, 1986). The neutral wind effect on AGWs is illustrated in Fig. 1e using a simulated wind profile $U_{x}(z)$ that includes a model tropospheric jet stream (Mastrantonio et al., 1976). When the AGW phase velocity is comparable to the wind velocity, the rays deviate significantly from the windless case (for $\alpha_{0}>84^{\circ}$ in Fig. 1). Ducting in a suitable wind profile may extend the rays to greater distances from the source although the gravity waves are absorbed in critical layers. On the other hand, AGWs encountering an opposing wind may be reflected. The refractive index and vertical wavelength become imaginary, indicating a reflection, but the associated evanescent wave can tunnel through the reflection layer, transferring energy below it. An over-reflection (reflection coefficient $>1$ ) may occur and result in wave amplification drawing energy from the wind.

\section{Pulsed ionospheric convection and traveling iono- spheric disturbances}

In the upper atmosphere, passing AGWs/TIDs modulate the ionospheric layers and this is observed as a modulation of the HF radar ground-scatter. SuperDARN can also observe the high-latitude sources of AGWs, namely the pulsed ionospheric convection which is a result of solar wind MIT coupling. Prikryl et al. (2005) studied TIDs for a case when the ionospheric convection was pulsed by solar wind Alfvén wave coupling to the dayside magnetosphere. These pulsed ionospheric flows (PIFs) that are observed by HF radars are ionospheric signatures of pulsed magnetic reconnection associated with flux transfer events at the dayside magnetopause (Provan et al., 1998) or pressure pulses in the magnetosheath (Lin et al., 1996). The radar ground-scatter signatures of TIDs are commonly observed equatorward of PIFs. A one-to-one correspondence between convection electric field enhancements and TIDs has been observed (Prikryl et al., 2005) and this is consistent with previous TID observations and modeling (e.g. Lewis et al., 1996; Millward et al., 1993a, b). As shown in Sect. 3, the gravity wave energy from an auroral source in the E-region ionosphere not only reaches the F-region directly or via reflection in the upper mesosphere or lower thermosphere but also penetrates to the lower atmosphere where it can be reflected back to the ionosphere. In this section we present observations of TIDs and their sources in the auroral ionosphere.

\subsection{Case 1: 2 November 1999}

Disturbed conditions in the upstream solar wind were caused by the arrival of a moderate corotating plasma stream from a coronal hole. The compressional solar wind fluctuations in the interaction region at the leading edge of the stream were followed by Alfvén waves that modulated the dayside 
ionospheric convection that was a source of gravity waves (Prikryl et al., 2005). Figure 2 shows the Kapuskasing radar power at ranges $900-1900 \mathrm{~km}$, which correspond to $66^{\circ}-$ $74^{\circ}$ magnetic latitude. The radar echoes were mainly due to ground scatter except for the last $1-2 \mathrm{~h}$ when the ground scatter was mixed with ionospheric scatter. The slanted bands of strongly focused ground scatter power are due to equatorward-moving TID fronts in the ionosphere.

The time series of the Upernavik (UPN) ground magnetic field (northward) X-component is shifted by $10 \mathrm{~min}$ to approximately account for TID propagation from a source in the ionospheric footprint of the magnetospheric cusp just poleward of the ground scatter maximum range. The arrows in Fig. 2 mark a sequence of TIDs associated with the UPN $\mathrm{X}$-component fluctuations showing a one-to-one correspondence.

Figure 3 shows a sequence of the Kapuskasing radar scatter maps displaying the ground-scatter signatures of two successive TIDs. The ground scatter band 2 spanning the radar field of view at an angle approximately 30 degrees from the geographic east-west direction moved equatorward while the band 3 appeared about $400 \mathrm{~km}$ poleward of it. A band of ionospheric scatter in the TID source region appears at far ranges (near $80^{\circ}$ magnetic latitude).

The convection and potential maps (Fig. 4) are constructed using the line-of-sight velocity data from all SuperDARN radars. The fitted convection velocity vectors are shown color-coded (see velocity scale bar top-left). The cross-polarcap electric potential contour map is shown by solid and broken lines outlining the dusk and dawn convection cells, respectively. The value of the cross-polar-cap voltage is printed in the upper-left corner. The cross-polar-cap potential was moderately enhanced after the onset of dayside reconnection due to the IMF southward turning. The IMF $B_{Z}$ versus $B_{Y}$ clock-dial plot is shown (top-right).

TID 2 was generated by a convection pulse shown by red velocity vectors around $12 \mathrm{~h}$ of magnetic local time (MLT) (Fig. 4). The convection pulse forms a flow channel (Pinnock et al., 1991) that is an ionospheric signature of a flux transfer event due to magnetic reconnection between the IMF and the geomagnetic field.

The flow channel extended from Taloyoak (TAL; $78.5^{\circ}$ ) in Nunavut to Clyde River (CYR) on Baffin Island (79.7 magnetic latitude) in Canada, and to Upernavik (UPN) on the west coast of Greenland. The ground magnetic field pulsation observed under the eastern part of the flow channel at UPN was very similar to the pulsation observed $\sim 550 \mathrm{~km}$ westward at CYR, and was partly detected at TAL. The CYR pulsation (not shown) led the UPN pulsation in phase by $1-2 \mathrm{~min}$. This is expected for a footprint of newly reconnected magnetic flux tube moving north-eastward at a speed between 5 and $9 \mathrm{~km} / \mathrm{s}$. The ground magnetic field signatures at UPN and CYR are consistent with the current system associated with flux transfer events for IMF $B_{Y}<0$ (Øieroset et al., 1997, their Fig. 11). In such a case, the negative-to-

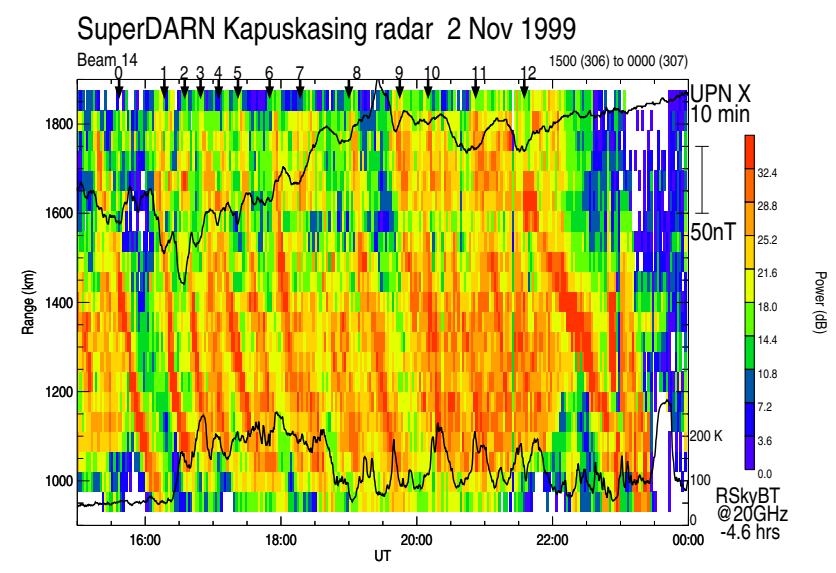

Fig. 2. The Kapuskasing radar ground scatter power modulated by passing TIDs. The UPN ground magnetic field $\mathrm{X}$ component is shifted by $10 \mathrm{~min}$. The arrows mark the TIDs that are associated with UPN-X fluctuations. The radiometric sky brightness temperature observed from Ottawa (magnetic latitude $56^{\circ}$ ) is shifted by $4.6 \mathrm{~h}$.

positive deflections of the $\mathrm{X}$ component are caused by a reversal of the ionospheric Hall currents from westward to eastward as the current system shifts poleward. Such east-west ionospheric currents (convection flow channels) are sources of AGWs/TIDs with wave fronts that can extend over more than $3000 \mathrm{~km}$ (Bristow and Greenwald, 1996).

In summary, there is a one-to-one correspondence between negative-to-positive deflections (see arrows in Fig. 2) of the ground magnetic field $\mathrm{X}$ component at UPN and the TID ground-scatter signatures. The perturbations of the ground magnetic field are due to auroral electrojet intensifications and the associated pulsed ionospheric flows (PIFs) that are a source of AGWs/TIDs. Thus the auroral AGW/TIDs are a consequence of solar wind MHD wave coupling to the magnetosphere.

Large amplitude fluctuations of the radiometric sky brightness temperature (RSBT) that are due to passing cloud/rain bands above Ottawa are shown superposed in Fig. 2. This time series is shifted by $4.6 \mathrm{~h}$ and the correlation results are discussed in Sect. 5.

\subsection{Case 2: 17 November 2001}

The corotating solar wind plasma stream from a coronal hole reached the earth's magnetosphere on 15 November (Prikryl et al., 2009, their Fig. 1). The solar wind Alfvén waves that coupled to the dayside magnetopause modulated the magnetic reconnection. The ionospheric signatures of it were observed by SuperDARN in the pre-noon sector. The convection/potential map (Fig. 5a) shows a remnant of one of the flow bursts north of Svalbard near 10:00 MLT that occurred a few minutes earlier at 05:20 UT. Associated with the dayside PIFs (reconnection signatures) were intense 


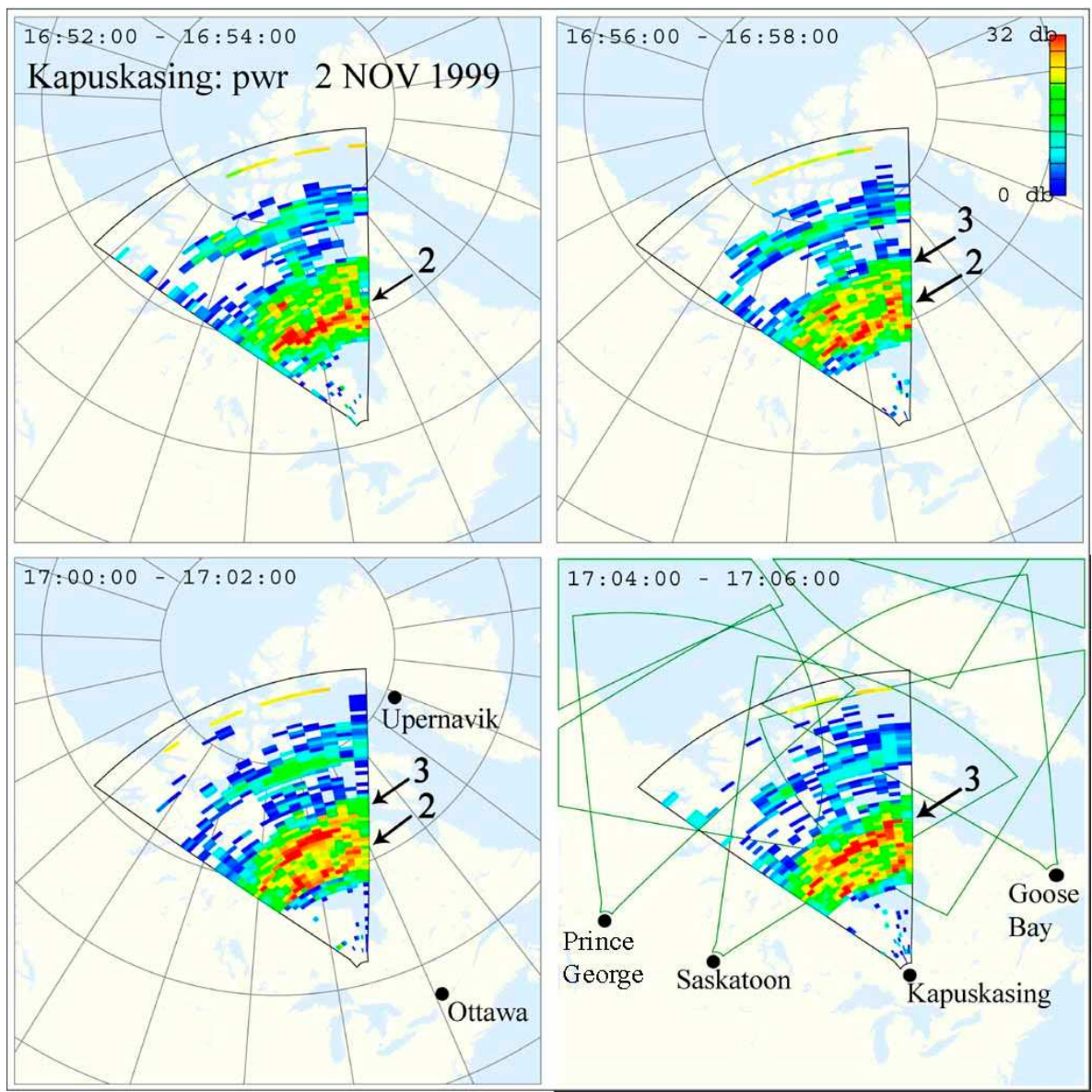

Fig. 3. The ground scatter signatures of a pair of equatorward-moving TIDs (labeled 2 and 3) observed by the Kapuskasing radar. A band of ionospheric scatter at far ranges is the TID source region. The SuperDARN radar fields of view, the Greenland magnetometer site at Upernavik and the radiometer site in Ottawa are shown. The geomagnetic latitude/longitude grid is superposed.

flow bursts in the dusk convection cell. They were caused by pressure pulses generated in the magnetosheath through the interaction of solar wind Alfvén waves with the bow shock (Lin et al., 1996). The magnetometer on board the geo-synchronous satellite GOES 10 observed quasi-periodic compressions of the magnetosphere that were associated with the intense convection pulses in the evening sector (Fig. 5b). The compressions modulated the ionospheric convection in the expanded dusk cell (IMF $B_{Y}>0$ ). This series of PIFs in the dusk convection cell over Alaska/Yukon is associated with a sequence of cloud bands (Sect. 5).

A sequence of three major convection pulses (A, B and C) was observed primarily by the Prince George radar beams looking from British Columbia over Alaska/Yukon (pulse B is shown in Fig. 5a). Figure 5b shows negative (away from the radar) line-of-sight velocity for beam 4 . The broken line shows the magnitude of the magnetic field observed by GOES 10 in the magnetosphere. A series of three compressions are correlated with the ionospheric convection pulses A-C. The pulse A that was observed at $\sim 04: 48$ UT
(Prikryl et al., 2003) was followed by pulses B at $\sim 05: 22$ UT (Fig. 5a) and $\mathrm{C}$ at $\sim 06: 00 \mathrm{UT}$. The ionospheric convection pulses A-C were preceded by quasi-periodic but weaker PIFs at higher latitudes (north of Alaska) before the dusk convection cell expanded to the size shown in Fig. 5a. These earlier PIFs were a source of equatorward propagating AGWs with a period $\sim 40$ min that caused TIDs observed by the radar in Kodiak, Alaska, in the ground scatter power before 04:00 UT (not shown). The three major ionospheric convection pulses A-C, and another one (D) on the night side of the dawn cell, that moved over northern Alaska at 11:10 UT (not shown) occurred too close to the radar for detection of the gravity waves (TIDs) that would ultimately have been seen equatorward of the source region. The flow axes of the convection pulses A$\mathrm{D}$ were tilted from the geographic east-west direction at an angle of about 20-30 degrees (azimuth of 290-300 ${ }^{\circ}$ ).

Ray tracing results (Fig. 1) suggest that the auroral AGWs would reach the troposphere at horizontal distances of $\sim 1000 \mathrm{~km}$ or more from the suggested source. A possible impact of these AGWs on tropospheric convection in an 


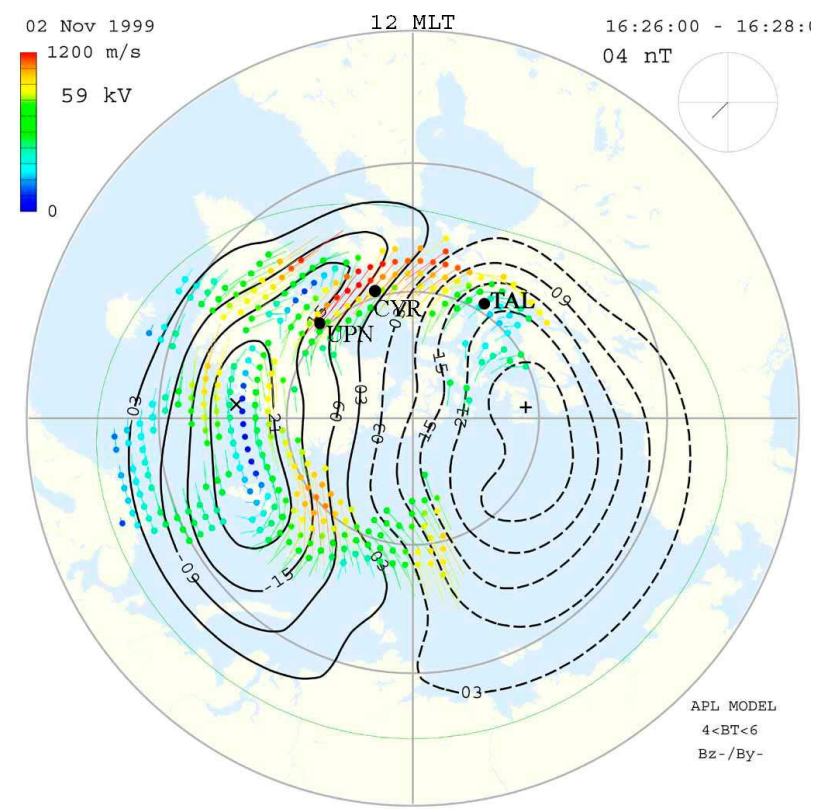

Fig. 4. Ionospheric convection flow channel centered at 12:00 MLT: a source of TID 2. Magnetometer locations near the cusp footprint are shown.

extratropical cyclone over the Gulf of Alaska is discussed in Sect. 5.2.

\section{Cloud bands in extratropical cyclones}

\subsection{Case 1: 2 November 1999}

The 20-s averaged radiometric sky brightness temperature (RSBT) at $20 \mathrm{GHz}$ is superposed in Fig. 2 (bottom) on the radar ground scatter power showing TIDs. The RSBT fluctuations were closely correlated with the attenuation of the ACTS satellite 27.5-GHz beacon signal (not shown) due to clouds or rain. The RSBT time series that is shifted by $4.6 \mathrm{~h}$ shows a striking similarity with the UPN magnetic field Xcomponent and TID time series. There is an almost one-toone correspondence between TIDs and/or the UPN-X pulses, and the RSBT peaks. The UPN-X ground magnetic field pulsations pertaining to the source of AGWs show an onset with a period $\sim 20 \mathrm{~min}$ that is later changing to $\sim 50 \mathrm{~min}$, which is very similar to RSBT fluctuations. The quasi-periodic variations of RSBT and the attenuation of the beacon signals (not shown) were caused by mesoscale cloud/rain bands passing over Ottawa (magnetic latitude $56^{\circ}$ ). The above time delay is comparable with or exceeds the possible downward group propagation times of AGWs launched by an E-region source (Fig. 1a and e).

Figure 6 shows the results of the time series analysis. The UPN X-component time series is shown (Fig. 6a, top) shifted by $\sim 5 \mathrm{~h}$ to match the onset of the RSBT fluctuations.
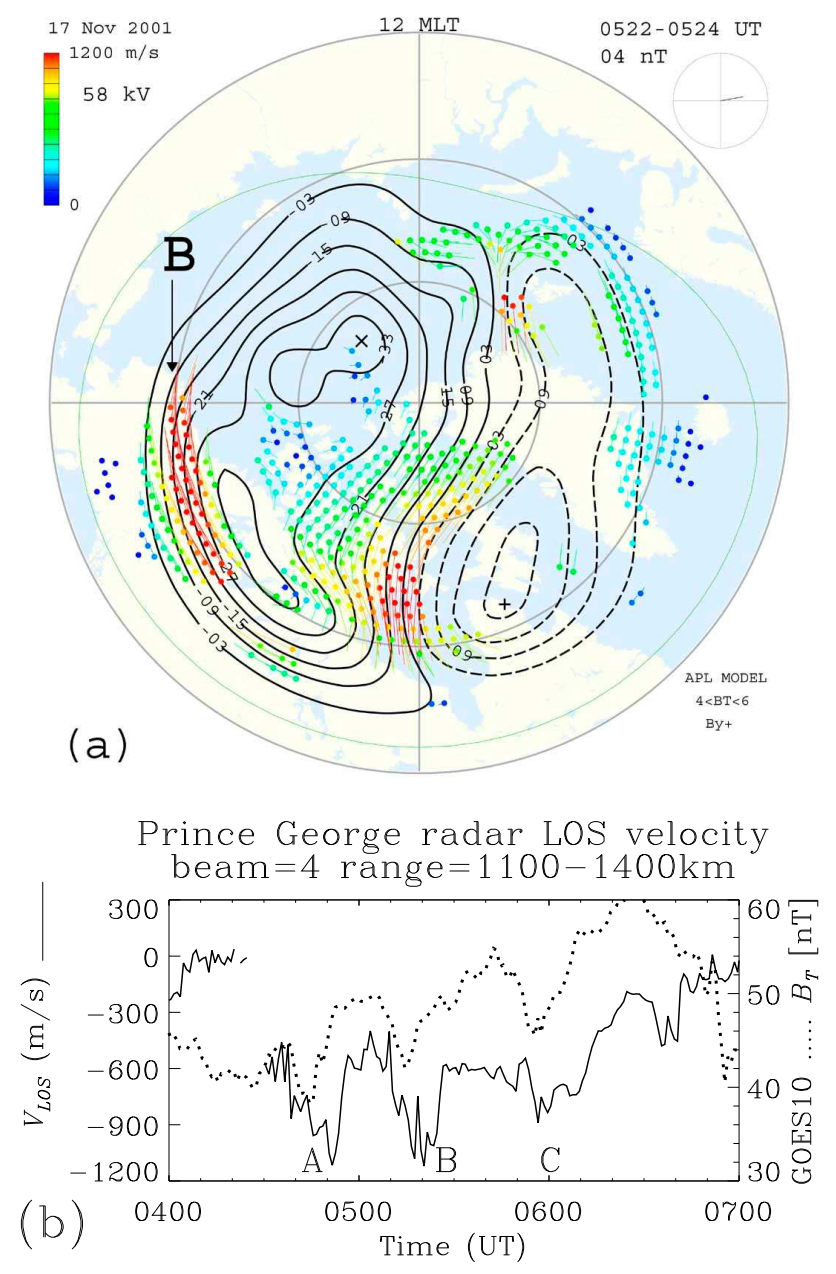

Fig. 5. (a) The convection/potential map showing an intense convection pulse B. (b) The Prince George radar line-of-sight velocity (solid line) and magnetic field magnitude (dotted line) at geosynchronous orbit.

Two time series of the TID-modulated radar ground scatter power, each being an average over three adjacent radar range gates centered at $\sim 1130$ and $1670 \mathrm{~km}$ for the Kapuskasing radar beam 14, are also shifted so that the strongest UPN $\mathrm{X}$-component negative pulse lines up with the sharpest TID band 2 and the strong RSBT peak observed at $\sim 21: 25$ UT as indicated by the yellow vertical band. These approximate relative shifts are then used in the cross-correlation analysis to represent lag 0 . There is an almost one-to-one correspondence between TIDs and RSBT enhancements as suggested in Fig. 6a.

Figure $6 \mathrm{~b}$ shows normalized fast Fourier Transform (FFT) spectra of the UPN X-component, the 20-GHz RSBT and SuperDARN ground-scatter power (TIDs). Low frequencies were filtered out by subtracting a one-hour running average before computing the FFT. For TIDs, the spectrum is an average of three spectra of TID time series (one intermediate range at $1360 \mathrm{~km}$ that is not shown in Fig. 6a is added to 

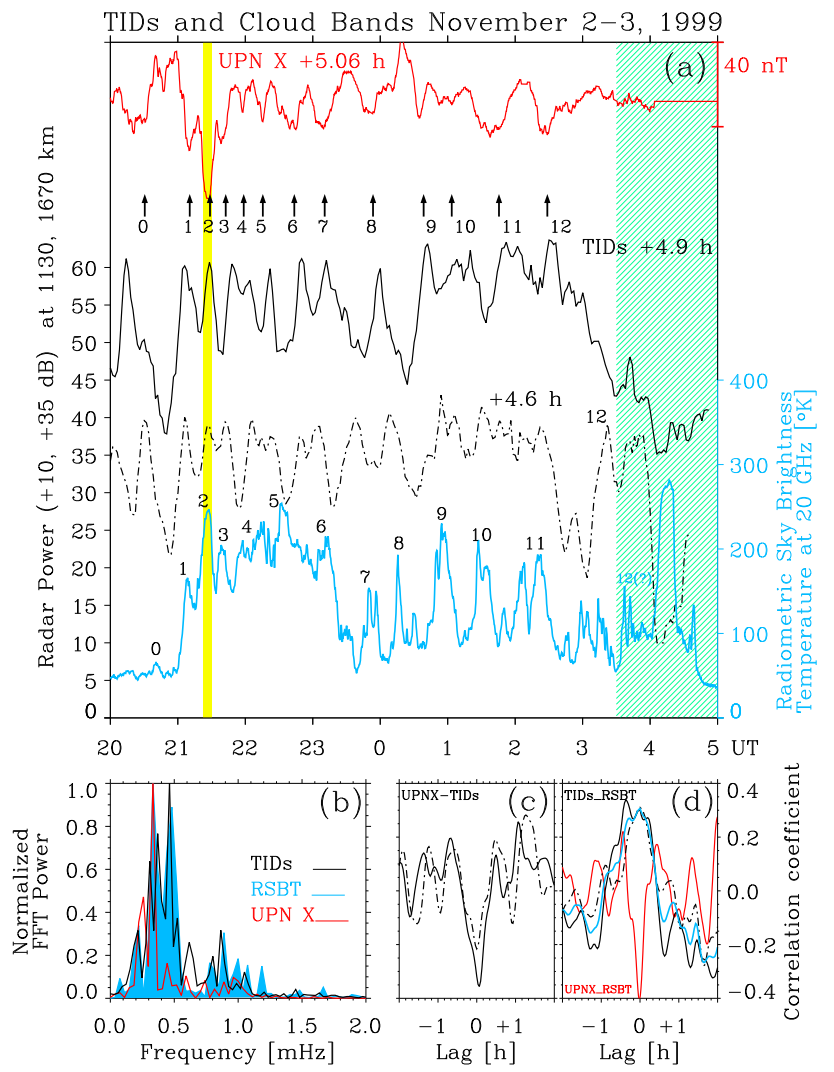

Fig. 6. The cross-correlation and spectral analysis of the ground magnetic field, TIDs and cloud/rain bands. (a) Time series of (top) UPN X-component and (middle) averaged radar ground scatter power (TIDs) at two ranges are shifted with respect to the radiometer data (bottom). The arrows are the same as in Fig. 2. The correspondence between TIDs/PIFs and the RSBT enhancements is suggested. (b) Normalized FFT spectra of detrended time series. (c-d) Cross-correlation analysis of pairs of time series excluding the last 90 min interval (hatched).

compute the average). All the spectra show two main bands of spectral power near $\sim 0.4$ and $0.9 \mathrm{mHz}$ (periods of $\sim 40$ and $20 \mathrm{~min}$ ). This similarity extends to some of the finer spectral structure (peaks) as well.

Figure $6 \mathrm{c}$ and $\mathrm{d}$ shows cross-correlation functions (XCFs) for pairs of time series computed for the time interval shown in Fig. 6a, excluding the last $90 \mathrm{~min}$ (hatched) when the ground scatter is mixed with the ionospheric scatter. The CYR X-component (not shown) was highly correlated with the UPN X-component (correlation coefficient +0.66 with a lag of $80 \mathrm{~s}$ ). This is consistent with observed east-west extended flow channels as discussed in Sect. 4.1. Thus the CYRX-RSBT XCFs (not shown) are very similar to those of the UPN X-component.

The UPN-X time series are anti-correlated with the ground scatter power due to TIDs. The XCFs for UPN-X data (Fig. 6c) show negative peaks centered near lag 0 that corre-

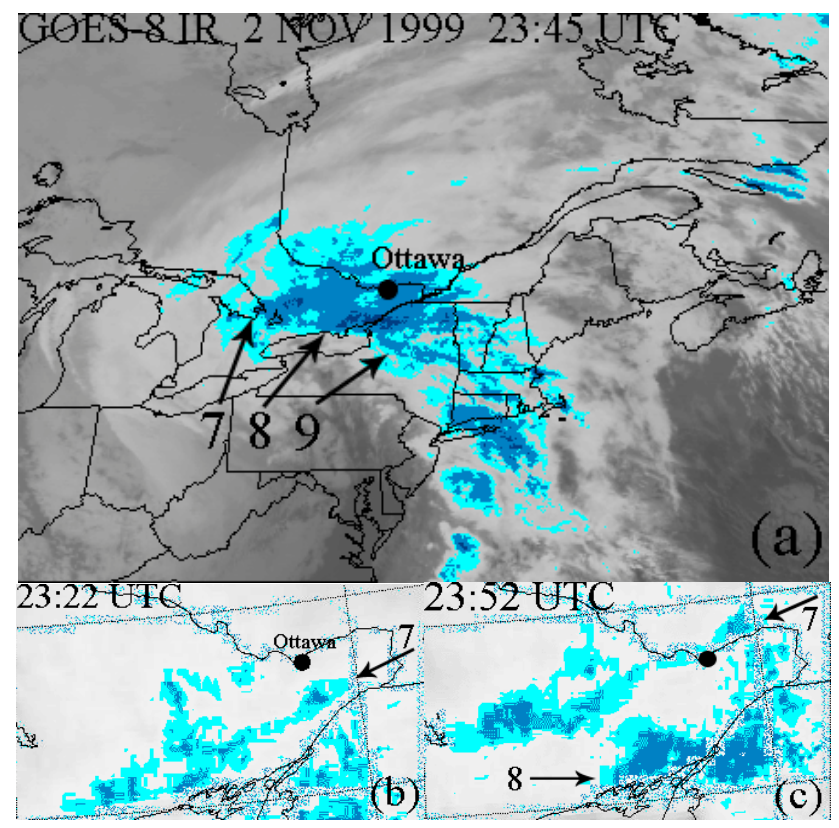

Fig. 7. GOES-8 infrared images showing cloud bands (numbers refer to RSBT enhancements in Fig. 6a) in a mid-latitude cyclone. Dark-blue coding is used to bring out low-contrast features and to highlight low cloud-top temperatures of convective cloud bands.

sponds to relative shifts introduced in Fig. $6 \mathrm{a}$ (10 and $28 \mathrm{~min}$ for UPN-X versus TIDs at 1670 and $1130 \mathrm{~km}$, respectively). The best correlation $(-0.36)$ is for TIDs at $1670 \mathrm{~km}$, which is near the source region of AGWs (PIFs).

The UPN-X time series are anti-correlated with the RSBT variations. The XCFs (Fig. 6d, red line) show correlation coefficients of -0.40 at a lag of $-1 \mathrm{~min}$ for UPN-X. This lag represents a small correction to the approximate time shifts in excess of $5 \mathrm{~h}$ that are used in Fig. 6a. The TIDs are correlated with the RSBT time series although the TID-RSBT XCFs (see the black solid and broken lines in Fig. 6d) are broader, with the highest correlation coefficient +0.34 . The mean XCF (shown by a blue line) peaked at +0.30 for lag 0 .

Figure 7a shows the GOES- 8 satellite infrared image of a mid-latitude cyclone that is structured by cloud bands. Low cloud-top temperatures are shown in shades of blue, with dark blue indicating the coldest cloud top level. The cloud bands formed in the warm frontal zone of the cyclone and moved poleward with the vortex flow. Bands 7 and 8 and a trace of band 9 that formed east of Lake Ontario are identified. High-resolution infrared (IR) images (Fig. $7 \mathrm{~b}-\mathrm{c}$ ) show the band 7 that started to form about two hours before it passed over Ottawa at $\sim 23: 52$ UT. The band is tilted from the east-west direction at an angle of 20-30 degrees, which differs from the tilt of the TID fronts (Fig. 3) by less than 10 degrees. The next band 8 moved over Ottawa $\sim 30$ min later. The strongest RSBT peak $(\sim 04: 15$ UT on 3 November) was due to a cold frontal band moving 
in the south-westerly direction (north-eastward). Although inconspicuous in satellite IR images, the band was prominent in the images obtained by the Environment Canada weather radars (not shown). The weather radars also observed the east-west oriented band-like forms moving poleward but clear rain bands were rarely resolved.

The warm sector and frontal zone are known as regions where moist gravitational and moist symmetric instabilities exist (Schultz and Schumacher, 1999, see their Fig. 6). While the air parcels approaching the warm front from within the warm zone may rise naturally over the colder air masses ahead of the warm front, an additional quasi-periodic lift could be provided by aurorally forced AGWs as discussed in Sect. 6.

Ray tracing of auroral AGWs in a stationary atmosphere model (Fig. 1) shows that downward AGW group rays reach the troposphere at a horizontal distance $\sim 1000 \mathrm{~km}$ or more from the assumed source in the E-region. The distance between the cusp footprint where PIFs were observed and Ottawa is $\sim 2000 \mathrm{~km}$. However, a wind profile encountered by AGWs can significantly modify the group path (Fig. 1e), extending it horizontally due to ducting. When reaching the instability region in the troposphere the gravity wave may initiate convection by providing lift to release the MSI, thus forming cloud bands (Sect. 6). Once the convection initiated, the cloud bands would move with the cyclonic flow and evolve into rain bands such as those that were observed by the radiometers in Ottawa.

\subsection{Case 2: 17 November 2001}

The GOES-10 IR enhanced images (Fig. 8) show a midlatitude cyclone that is structured by east-west oriented cloud bands appearing, deepening (the temperature decrease indicates the vertical growth of the cloud), and eventually fading.

As in Case 1, the formation of a triplet of bands A-C appears to have been initiated by gravity waves launched by the ionospheric convection pulses A-C (Fig. 5). The band $\mathrm{A}$ appeared first, moved poleward with the wind and was followed by bands B and C, each forming equatorward of the previous poleward-moving band, in sequence. Initially, the bands were tilted from the east-west direction at an angle of 10-30 degrees, which is similar to the tilt of the convection flow axis over northern Alaska. Once formed, the bands progressed poleward and were distorted by the counter-clockwise wind in the vortex. The head of the cyclone was already structured into weaker bands that appeared earlier and were sequenced like the ionospheric convection pulses just north of Alaska. No major cloud band of a similar scale size and height (temperature) formed following band $\mathrm{C}$ until $\sim 16: 00 \mathrm{UT}$, i.e. $\sim 5 \mathrm{~h}$ after band $\mathrm{C}$. This later cloud band D (shown extended towards Queen Charlotte Islands at 18:00 UT) was associated with another major ionospheric convection pulse at 11:10 UT, a likely source of a large-amplitude gravity wave (Sect. 4).

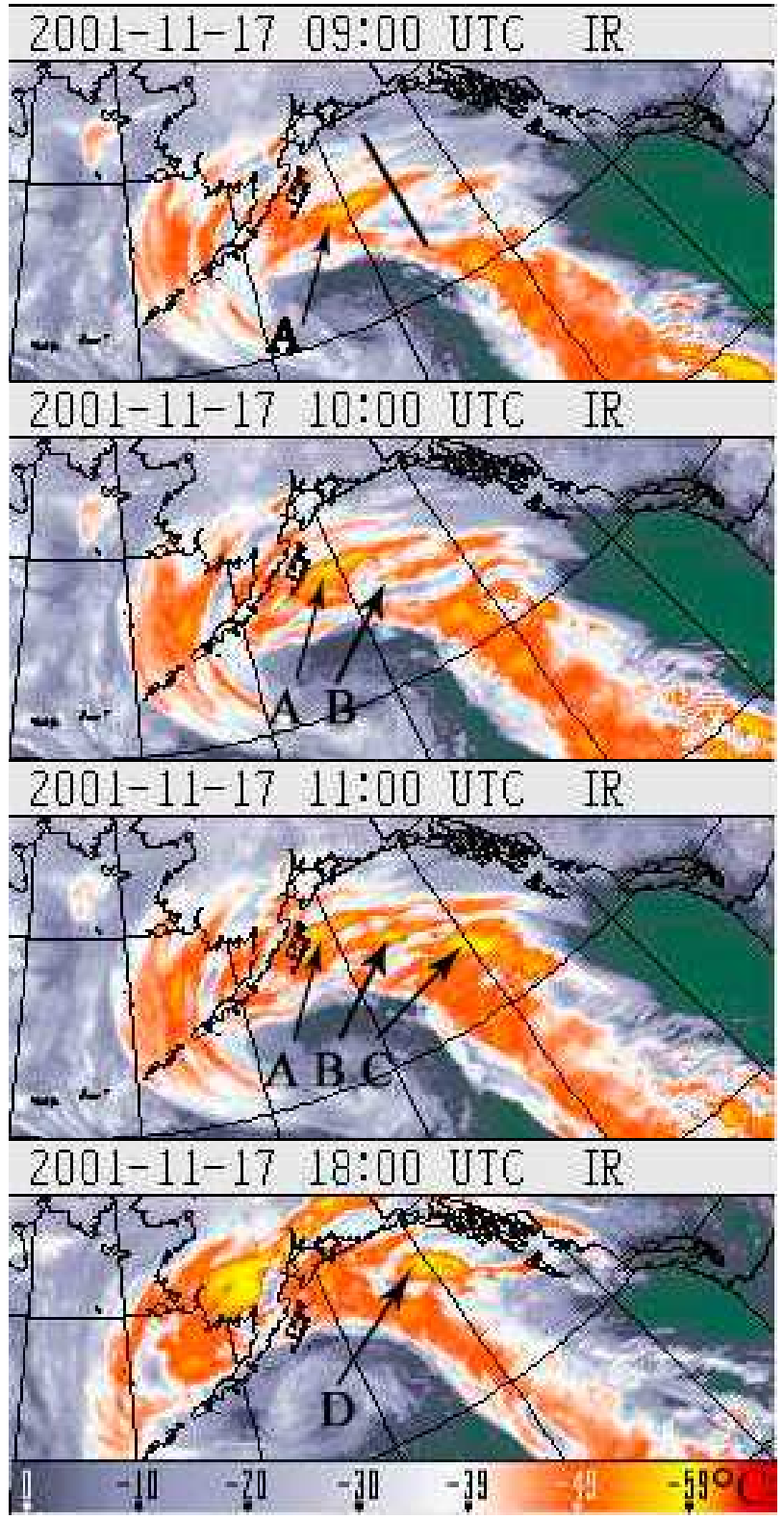

Fig. 8. The GOES-10 infrared images show cloud bands forming in the warm frontal zone of a mid-latitude cyclone. The bands A$\mathrm{C}$ formed in sequence (top three panels). The band D started to form about $5 \mathrm{~h}$ later. The heavy line segment (top panel) shows the location of the projection of a cross-section of troposphere that is considered in Fig. 9.

The ray tracing results of Fig. 1 for the auroral AGWs with a period of $40 \mathrm{~min}$ indicate that AGWs reach the troposphere at a horizontal distance $\sim 1000 \mathrm{~km}$ or more from an ionospheric source a few hours after being launched. This distance, which would be affected by the actual wind profile encountered by AGWs (Fig. 1e), is approximately the same as the distance of $\sim 1500 \mathrm{~km}$ between the AGW source 
SYMMETRIC INSTABILITY RELEASED BY A GRAVITY WAVE

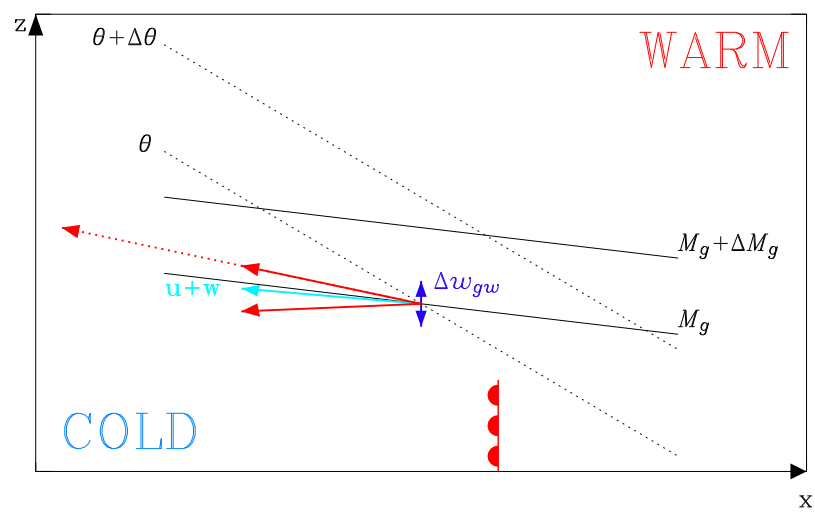

Fig. 9. Symmetric instability released by a gravity wave perturbation. The slopes of isolines of $\theta$ and $M_{g}$ in the x-z plane are such that $\partial \theta / \partial z>0$ and $\partial M_{g} / \partial x>0$, meaning that the atmosphere is stable to purely vertical and horizontal displacements. Slantwise displacements with intermediate slopes between the isolines of $\theta$ and $M_{g}$ are symmetrically unstable. A vertical perturbation $\Delta w_{g w}$ due to a gravity wave (dark blue) vectorially added to the upward tilting flow (light blue) in the vicinity of a warm front will release the MSI, thus leading to slantwise convection.

over northern Alaska and the cloud bands A-C over the Gulf of Alaska. The one-to-one correspondence between ionospheric convection pulses and the appearance of cloud bands suggests that the formation of the cloud bands was stimulated by auroral AGWs that were generated by the ionospheric convection enhancements observed over Alaska/Yukon.

As in Case 1, these cloud bands formed near the warm frontal zone where the moist symmetric instability (MSI) could have been present. It is suggested that the incoming AGWs provided the lift to release MSI and initiated slantwise convection (Sect. 6). It should be noted that only a fraction of the longitudinally extended wave front of an auroral AGW intercepting the instability region would be involved in the band formation.

\section{The symmetric instability}

The Bergen school introduced new concepts and constructed a cyclone model (Bjerknes, 1919, 1920; Bjerknes and Solberg, 1922) based on careful and extensive observations of cyclone-related weather that were at the forefront of meteorology at the time. Bjerknes (1920) observed rain stripes of broad and narrow types, stationary and moving stripes, a broad type followed by a narrow type. He correctly identified these bands with the structure of the atmosphere in the vicinity of the warm and cold fronts within the context of atmospheric circulation that is now referred to as the warm and cold conveyor belts. With remarkable clarity and simplicity, he described a concept of a cyclone and thus laid the foundation for the modern understanding of cyclones. Of par- ticular interest here is the mesoscale structure in the warm frontal zone (broad rain stripe), which Bjerknes likely could not resolve even with his impressive network of 90 stations. As mentioned above this is the region in extratropical cyclones where symmetric instabilities may result in formation of cloud/rain bands.

This section attempts to briefly summarize the concept of the symmetric instability focusing on some basic properties to support the proposed interpretation of the above observations (Sect. 5). It is suggested that auroral AGWs provide a trigger to release the instability and initiate the slantwise convection. For derivation and detailed discussion of the symmetric instability see, e.g. Bennetts and Hoskins (1979), Bluestein (1993), Houze (1993), Emanuel (1994), and Schultz and Schumacher (1999).

The dry (and moist) symmetric instability can be described as an interplay or simultaneous action of buoyancy and Coriolis restoring forces on a parcel of air in the vertical and horizontal directions, respectively. If a parcel of air is displaced upward in a buoyantly unstable environment (negative thermal stratification), it becomes buoyant and accelerates upward (buoyant/gravitational instability). Similarly, in an inertially unstable environment (negative horizontal shear), a parcel that is displaced in a horizontal direction obtains an excess of momentum away from the geostrophic equilibrium. When the atmosphere is stable for purely vertical and purely horizontal displacements but nonetheless is unstable to sloping or slantwise displacements, the condition is called symmetric instability. The word "symmetric" here implies that the basic state and perturbations are independent of one of the two horizontal coordinates (the y-coordinate is chosen below). In the development of the theory of symmetric instability, an air parcel taking the form of an infinitely long tube is assumed. It may be noted that if such symmetry is not applicable, one needs to consider the more general problem of baroclinic instability, of which the symmetric instability is a type.

The theory of symmetric instability is applicable for twodimensional flows. We assume an X-Z-plane similar to one used in 2-D ray tracing (Sect. 3) but with the x-coordinate increasing perpendicularly to a warm front towards warmer air. In other words, a cross-section of the atmosphere is considered that is perpendicular to cloud bands (Fig. 8; top panel). Using parcel theory and assuming an inviscid, geostrophically and hydrostatically balanced mean state, the geostrophic absolute momentum is $M_{g}=v_{g}+f x$, where $v_{g}$ is the geostrophic wind in the y-direction and $f$ is the Coriolis parameter. The condition for the buoyant (gravitational) instability is $\partial \theta / \partial z<0$, where $\theta$ is the potential temperature and $z$ is the vertical coordinate. The condition for the inertial instability is $\partial M_{g} / \partial x<0$. The condition for the dry symmetric instability is illustrated by the so-called $\left(M_{g}, \theta\right)$ relationship in Fig. 9. A parcel is inertially stable to purely horizontal displacements $\left(\partial M_{g} / \partial x>0\right)$ and gravitationally stable to purely vertical displacements $(\partial \theta / \partial z>0)$. If the surfaces of constant 
$\theta$ slope more steeply than the surfaces of constant $M_{g}$ then any slantwise displacement between the $M_{g}$ and $\theta$ surfaces is symmetrically unstable and the parcel will continue to accelerate farther away from its equilibrium position.

Schultz and Schumacher (1999) detailed many complications to the theory of moist symmetric instability, distinguishing between the potential symmetric instability (PSI) and the conditional symmetric instability (CSI). In the above discussion of dry symmetric instability, the potential temperature $\theta$ should be replaced by the equivalent potential temperature $\theta_{e}$ for PSI and by the saturation equivalent temperature $\theta_{e}{ }^{*}$ for CSI. If the air is moist but unsaturated, conditional instability (CI) can exist. It occurs locally where the environmental lapse rate lies between the moist- and dry-adiabatic lapse rates, or $\partial \theta_{e} * / \partial z<0$ (Schultz and Schumacher, 1999). For the definition of $\theta_{e}$ and $\theta_{e}^{*}$ (sometimes denoted as $\theta_{e s}$ ), the reader is referred to Houze (1993). The CSI condition occurs when the temperature lapse rate of moist but unsaturated air is conditionally unstable on a surface of constant $M_{g}$ (i.e. the slope of $M_{g}$ surfaces is less than the slope of $\theta_{e}$ * surfaces).

An ingredients-based methodology for slantwise convection (reviewed by Schultz and Schumacher, 1999) requires three ingredients to produce deep, moist convection, namely instability, moisture and lift, thus clearly separating the instability from the lifting mechanism. All three ingredients must be present for slantwise convection to occur. The release of MSI is predicated upon slantwise parcel lifting, which can arise from synoptic or mesoscale processes, but the relative roles of these processes in the initiation of gravitational and slantwise convection are not well understood (Schultz and Schumacher, 1999). While mesoscale models are able to predict the banded structure in extratropical cyclones (Bennetts and Hoskins, 1979), gravity waves are known to produce some mesoscale bands. Auroral gravity waves have not been considered. It is argued that they may be a contributing factor in initiating the convection, particularly when the instability is near the threshold condition to be released by a small perturbation.

A few hours after the HF radars observed TIDs and their sources in the auroral ionosphere, observations of mesoscale cloud bands in mid-latitude cyclones indicate that the auroral AGWs have modulated the tropospheric clouds/precipitation. In Case 1, there is a close one-to-one correspondence between the sources of AGWs in the ionosphere (i.e. PIFs and/or ionospheric current fluctuations), TIDs, the enhancements of radiometric sky brightness temperature (RSBT) and satellite beacon signal fades due to cloud/precipitation bands in the warm frontal zone of a mid-latitude cyclone. Likewise, Case 2 shows a series of intense pulses of ionospheric convection (a source of AGWs) associated with a sequence of cloud bands that formed near the warm front of another rapidly growing cyclone.

As discussed in Sect. 1.2, at tropospheric altitudes, the auroral gravity waves are expected to have amplitudes that have been reduced to values, which are comparable to mean vertical motions in the troposphere but which are smaller than typical instantaneous vertical motions, unless amplified. Although the direct impact of ionospherically generated AGWs on tropospheric weather may seem energetically implausible, amplification of the incident auroral AGWs is a distinct possibility, because of likely over-reflection from the wind shear associated with the MSI. However, small perturbations due to these gravity waves may trigger release of tropospheric instabilities that are very near the threshold. In particular, a small lift exerted on a parcel of air by a gravity wave in the presence of an upward tilting flow in the vicinity of the warm front, may initiate the slantwise convection (Fig. 9). Vertical velocity amplitudes of a few $\mathrm{cm} / \mathrm{s}$ applied over a fraction of the gravity wave period may contribute to a significant lift of the unstable layer. For example, an upward vertical motion due to an auroral gravity wave amplitude of $5 \mathrm{~cm} / \mathrm{s}$ over $10 \mathrm{~min}$ would result in a vertical displacement of an air parcel by $30 \mathrm{~m}$. If the air flow near the warm front is tilted upward at an angle that is less than but nearly equal to the slope of the $M_{g}$ surfaces, only a small perturbation due to a gravity wave is needed to release the MSI. In this way, incident auroral AGWs could modulate the slantwise convection, leading to precipitation and latent heat release, which in turn can result in rapid development and intensification of extratropical cyclones (e.g. Kuo and Low-Nam, 1990).

\section{Solar wind streams and high-level cloud area index (HCAI)}

High-level clouds, primarily deep convective clouds in extratropical and tropical cyclones, as well as the intertropical convergence zone (ITCZ), account for a major fraction of precipitation. Apart from determining the weather, they play many roles in the climate system (Stewart et al., 1998), e.g. contributing to radiative forcing. However, clouds are poorly parametrized in climate models (Pallé et al., 2006). From a viewpoint of a climate modeler or weather forecaster, a detailed understanding of both the internal and external factors that affect the development of these cloud systems, particularly on sub-synoptic scales (mesoscale), is required for accurate modeling and forecast. This is because the mesoscale structure significantly affects the larger (synoptic) scale development (Stewart et al., 1998, and references therein).

If solar-wind-generated auroral AGWs initiate deep convection, as suggested in Sects. 5 and 6, they contribute to cloud formation. To test this conjecture we analyzed time series of high-level infrared cloud cover extracted from the ISCCP-D1 dataset. While the high-level cloud type (cloud top pressure $<440 \mathrm{mb}$ ) includes cirrus and cirrostratus in addition to deep convective clouds, the high-level cloudiness has been shown to be closely associated with extratropical cyclones. To trace the variation in the extent of high-level cloud in a given geographical sector, $280 \times 280 \mathrm{~km}^{2}$ cells are 
selected for which the high-level fractional cloud amounts exceeded an arbitrary threshold of 0.1 .

A high-level cloud area index (HCAI) is defined as the percentage ratio of the number of cells with above-threshold fractional cloud amounts $(>0.1)$ to the total number of cells for which the cloud data are available in a given geographical sector. Using the ISCCP-D1 dataset, the HCAI time series are constructed for various geographic sectors. Because of lower quality and sparsity of cloud estimates at very high latitudes, we limit the analysis to the latitude range from $-70^{\circ}$ to $+70^{\circ}$.

\subsection{Corotating stream interaction regions}

Prikryl et al. (2009) verified the relationship between solar magnetic sector boundary passage and tropospheric vorticity (Wilcox et al., 1974) that most likely relates to synoptic scales. It is noted that a corotating interaction region (CIR) at the leading edge of a solar wind stream is a more accurate marker of auroral activity onset than the sector boundary, which may differ by 1-2 days from the stream interface. Also, a sector boundary does not necessarily occur for every solar wind stream, nor is a sector boundary crossing necessarily followed by a significantly enhanced solar wind stream. Although usually there is a sector boundary between two adjacent major solar wind streams emanating from two consecutive coronal holes. As suggested in Section 5, by generating auroral AGWs, CIRs at the leading edge of streams and solar wind Alfvén waves may impact the mesoscale structure and growth of extratropical cyclones. In the companion paper (Prikryl et al., 2009) it is found that significant extratropical storms tend to occur within a few days of the arrival of CIRs or coronal mass ejections (CMEs).

As a consequence of solar rotation, a high-speed solar wind stream from a coronal hole generates a CIR, an interface between the fast and slow solar wind. Characteristics of CIRs in terms of solar wind parameters, namely solar wind velocity $V$, plasma (number) density $n_{p}$, magnetic field magnitude $|\mathbf{B}|$ and temperature are briefly described by Prikryl et al. (2009). These plasma parameters are used to identify the stream interface. Finding the characteristic peaks of $n_{p}$ and $|\mathbf{B}|$ at the leading edge of rising solar wind velocity $V$ (three parameter signature) is adopted as the principal method. The standard deviation $\sigma_{B z}$ of the IMF $B_{Z}$ component also peaks at the stream interface and remains elevated for a few days. This is then corroborated by coincident bipolar east-west deflections of the solar wind flow (Gosling et al., 1978). In the geocentric solar ecliptic (GSE) coordinates, the flow angle $\phi_{V}$ increases from zero for $-X_{\mathrm{GSE}}$ and is positive towards $+Y_{\mathrm{GSE}}$. The flow signatures are used to determine the arrival time when other signatures have been less clear, particularly when there are missing magnetic field data.

A total of 624 streams (CIRs) with a maximum velocity $V_{\text {MAX }}$ of $500 \mathrm{~km} / \mathrm{s}$ or more were identified in the period from July 1983 through June 2006 (Table 1). Because of solar wind data gaps prior to 1995 , not all of stream interfaces could be identified solely from solar wind data. However, if solar wind data gaps prevented identification of the stream interface (CIR) at the leading edge of a partially observed stream, geomagnetic indices (AE and/or $K_{p}$ ) are used as proxies when possible (72 cases). An onset of continuous and often recurrent geomagnetic activity caused by high-speed streams (Lundstedt, 1984; Tsurutani et al., 1987; McPherron and Weygand, 2006), which are preceded by geomagnetically quiet periods, provides a good proxy for stream arrival time. However, corotating streams are sometimes disrupted by CMEs, particularly near the solar maximum.

Arrival times of co-rotating streams are used as key times in the superposed epoch (SPE) analysis of solar wind plasma parameters (Fig. 10a) to show the mean variations of the parameters as sampled by a spacecraft crossing CIRs. The mean solar wind velocity decreases to a minimum just before the key time and then steeply rises to a maximum two days later. All other mean solar wind parameters peak at, or very close to, the key time. The mean flow angle $\phi_{V}$ shows a bipolar east-west deflection.

It is advantageous to combine some of the parameters in a single variable that would characterize the solar wind geoeffectiveness and correlate with solar activity indices. Osherovich et al. (1999) suggested a quasi-invariant in$\operatorname{dex} \mathrm{QI} \equiv\left(B^{2} / 8 \pi\right) /\left(\rho V^{2} / 2\right)=M_{A}^{-2}$, where $\rho$ is plasma density (protons in the first approximation), $M_{A}$ is the magnetic Mach number $\left(M_{A}=V / V_{A}\right.$, where $V_{A}$ is the Alfvén speed) and $B=|\mathbf{B}|$. The index is equivalent to the ratio of the solarwind magnetic to ram pressures, or the inverse of the magnetic Mach number squared. This non-dimensional quantity is of fundamental importance for describing the solar wind. Figure 10b shows the results of the SPE analysis of QI, AE (1983-1988 and 1990-1994) and $K_{p}$ indices. The mean QI and geomagnetic activity indices steeply rise to a maximum at the key time and decay a few days later. The maximum is preceded by low values of geomagnetic activity and QI, which is consistent with the finding by Borovsky and Steinberg (2006). In agreement with previous studies, on average, the mean AE-index is less than 200 nT for more than 2 days before the arrival of high-speed streams. As noted by Borovsky and Steinberg (2006), the calm intervals are characterized by low solar wind $V$, weak $B$, absence of Alfvén waves and a tendency for $B_{Z}$ to be northward.

The IMF $B_{Z}$ component (in geocentric solar magnetospheric (GSM) coordinate system) controls the solar wind coupling to the magnetosphere and the amount of joule heating energy deposition in the auroral ionosphere. The IMF $B_{Z}$ changes sign frequently, particularly when large amplitude Alfvén waves are present in the solar wind. At the mean stream interface (day 0 in Fig. 10a) the mean IMF $B_{Z}$ shows a small but statistically significant shift from positive (northward) to negative (southward) values, suggesting a marginal preponderance of CIRs associated with the southward $B_{Z}$ turning and a tendency for $B_{Z}$ to be northward during the 
Table 1. List of times (yymmddhh) of high-speed plasma streams arrivals used in the SPE analysis.

\begin{tabular}{|c|c|c|c|c|c|c|c|}
\hline 83071212 & 86012323 & 89101616 & 93050606 & 95122424 & 99012020 & 02030404 & 04042020 \\
\hline 83072323 & 86022020 & 89102929 & 93051212 & 96010202 & 99021111 & 02033030 & 04050404 \\
\hline 83080202 & 86031818 & 89112626 & 93052727 & 96011313 & 99030101 & 02041111 & 04051818 \\
\hline 83081212 & 86032121 & 89122222 & 93060303 & 96011919 & 99031212 & 02042727 & 04052828 \\
\hline 83081919 & 86042222 & 90010808 & 93061010 & 96012828 & 99032929 & 02052727 & 04061313 \\
\hline 83082929 & 86053030 & 90012020 & 93062323 & 96021010 & 99040404 & 02060808 & 04062828 \\
\hline 83090808 & 86062727 & 90012828 & 93070606 & 96021616 & 99041010 & 02061919 & 04071111 \\
\hline 83091515 & 86072424 & 90020404 & 93072020 & 96022222 & 99042626 & 02070505 & 04071616 \\
\hline 83092525 & 86080303 & 90021515 & 93080404 & 96030909 & 99050606 & 02070909 & 04081010 \\
\hline 83100404 & 86082020 & 90031818 & 93081515 & 96031616 & 99051313 & 02071212 & 04083131 \\
\hline 83101313 & 86091010 & 90041010 & 93082727 & 96032424 & 99051818 & 02071616 & 04100808 \\
\hline 83101717 & 86091717 & 90042222 & 93090202 & 96040808 & 99052323 & 02080909 & 04101919 \\
\hline 83102121 & 86092323 & 90050202 & 93091212 & 96041414 & 99060808 & 02082626 & 04110707 \\
\hline 83102828 & 86100505 & 90051010 & 93092929 & 96041717 & 99062626 & 02091111 & 04111919 \\
\hline 83110202 & 86101313 & 90053030 & 93100808 & 96051313 & 99072020 & 02091616 & 04112424 \\
\hline 83110707 & 86101818 & 90060606 & 93102626 & 96061515 & 99073030 & 02093030 & 04112929 \\
\hline 83111515 & 86111010 & 90071919 & 93110303 & 96070202 & 99081616 & 02100707 & 04120505 \\
\hline 83112828 & 86111515 & 90080101 & 93111818 & 96072828 & 99082323 & 02101414 & 04121616 \\
\hline 83120404 & 86112323 & 90081414 & 93112525 & 96081414 & 99090707 & 02102424 & 04122121 \\
\hline 83121010 & 86122121 & 90083030 & 93120101 & 96082828 & 99091212 & 02110101 & 04122525 \\
\hline 83123030 & 87020606 & 90090404 & 93121616 & 96091010 & 99092222 & 02111010 & 05010101 \\
\hline 84012828 & 87022020 & 90091111 & 93123131 & 96091919 & 99092626 & 02112121 & 05011212 \\
\hline 84022323 & 87030505 & 90100303 & 94011111 & 96092626 & 99100404 & 02112626 & 05012828 \\
\hline 84030101 & 87031818 & 90100909 & 94012626 & 96100202 & 99101010 & 02120606 & 05020606 \\
\hline 84030606 & 87032121 & 90102929 & 94020606 & 96100707 & 99102121 & 02121414 & 05022323 \\
\hline 84031717 & 87032525 & 90112727 & 94021818 & 96101111 & 99110707 & 02121919 & 05030606 \\
\hline 84032121 & 87040404 & 90120404 & 94030202 & 96101717 & 99111616 & 02122727 & 05032424 \\
\hline 84032828 & 87061111 & 91012424 & 94030606 & 96102222 & 99120202 & 03010303 & 05040404 \\
\hline 84042020 & 87071515 & 91021919 & 94032121 & 96102727 & 99122424 & 03011717 & 05041111 \\
\hline 84042525 & 87081212 & 91030404 & 94040202 & 96111313 & 99123030 & 03012222 & 05042020 \\
\hline 84050909 & 87082525 & 91032121 & 94050101 & 96112424 & 00011010 & 03012929 & 05043030 \\
\hline 84051919 & 87083131 & 91033030 & 94051414 & 96120202 & 00012727 & 03020404 & 05050707 \\
\hline 84060303 & 87090606 & 91042424 & 94052424 & 96120909 & 00020505 & 03021313 & 05052828 \\
\hline 84060808 & 87091010 & 91052222 & 94052828 & 96121515 & 00022323 & 03022020 & 05060404 \\
\hline 84061515 & 87101111 & 91061717 & 94061010 & 97010707 & 00032222 & 03022626 & 05061212 \\
\hline 84062929 & 87102222 & 91070808 & 94061919 & 97012626 & 00041616 & 03030303 & 05062323 \\
\hline 84071313 & 87110202 & 91071616 & 94062626 & 97020808 & 00051313 & 03031111 & 05070101 \\
\hline 84073131 & 87111010 & 91080101 & 94071414 & 97022121 & 00052222 & 03032727 & 05070909 \\
\hline 84081414 & 87112323 & 91081414 & 94072727 & 97022626 & 00052929 & 03033030 & 05072020 \\
\hline 84082323 & 87120303 & 91083030 & 94081010 & 97031212 & 00060303 & 03040808 & 05072727 \\
\hline 84082727 & 87120909 & 91090909 & 94082424 & 97032121 & 00061414 & 03041414 & 05080505 \\
\hline 84090808 & 87121515 & 91092525 & 94090505 & 97032828 & 00062222 & 03042121 & 05081313 \\
\hline 84091919 & 88010202 & 91100606 & 94100202 & 97041010 & 00080505 & 03042929 & 05082121 \\
\hline 84092323 & 88011212 & 91111313 & 94101313 & 97041616 & 00082828 & 03050505 & 05083131 \\
\hline 84100707 & 88020909 & 91112121 & 94102323 & 97050101 & 00091515 & 03051111 & 05090909 \\
\hline 84101919 & 88030808 & 91112828 & 94102929 & 97051515 & 00092424 & 03052121 & 05093030 \\
\hline 84110101 & 88032525 & 91120808 & 94110909 & 97062727 & 00101111 & 03052727 & 05100808 \\
\hline 84111515 & 88040101 & 91121616 & 94111919 & 97081313 & 00102222 & 03060202 & 05102525 \\
\hline 84121111 & 88042222 & 91122727 & 94112626 & 97090303 & 00110404 & 03060606 & 05110202 \\
\hline 84122626 & 88050505 & 92011212 & 94120505 & 97090808 & 00112424 & 03061414 & 05112929 \\
\hline 85010808 & 88051717 & 92020101 & 94121515 & 97092727 & 00120707 & 03061818 & 05121010 \\
\hline 85012727 & 88053030 & 92020808 & 94122020 & 97102323 & 01012121 & 03062626 & 05121919 \\
\hline 85020505 & 88062929 & 92022020 & 94122424 & 97112222 & 01020606 & 03070202 & 05122727 \\
\hline 85022727 & 88071111 & 92030808 & 95010202 & 98010606 & 01021313 & 03071111 & 06011515 \\
\hline 85030404 & 88080909 & 92031616 & 95011717 & 98020808 & 01022727 & 03072626 & 06012323 \\
\hline 85032828 & 88082020 & 92040303 & 95012929 & 98031010 & 01041818 & 03080606 & 06021010 \\
\hline 85040101 & 88083131 & 92050909 & 95021111 & 98032020 & 01050707 & 03082121 & 06021515 \\
\hline 85041919 & 88091010 & 92062323 & 95022626 & 98032424 & 01052323 & 03082929 & 06021919 \\
\hline 85062424 & 88091717 & 92072121 & 95030909 & 98041616 & 01060101 & 03090101 & 06030909 \\
\hline 85070404 & 88100505 & 92073030 & 95032626 & 98042323 & 01060808 & 03090808 & 06031414 \\
\hline 85071111 & 88101515 & 92080404 & 95040707 & 98043030 & 01061818 & 03091616 & 06031818 \\
\hline 85071717 & 88110202 & 92082020 & 95042222 & 98051616 & 01071515 & 03092424 & 06040909 \\
\hline 85072323 & 88111212 & 92090202 & 95042626 & 98052929 & 01072424 & 03100202 & 06041313 \\
\hline 85073030 & 88112525 & 92091414 & 95050202 & 98060505 & 01073131 & 03100505 & 06042121 \\
\hline 85081212 & 88113030 & 92092828 & 95051616 & 98061919 & 01081212 & 03101313 & 06050303 \\
\hline 85081818 & 88121111 & 92101111 & 95052323 & 98070202 & 01081717 & 03102424 & 06051111 \\
\hline 85082525 & 88122525 & 92102727 & 95053030 & 98071616 & 01082121 & 03110808 & 06051818 \\
\hline 85090808 & 89010404 & 92110909 & 95061919 & 98072121 & 01082525 & 03112020 & 06053030 \\
\hline 85091414 & 89012020 & 92120707 & 95062525 & 98082222 & 01090303 & 03113030 & 06060606 \\
\hline 85091919 & 89013131 & 93010202 & 95071616 & 98100101 & 01091111 & 03120505 & 06061414 \\
\hline 85100505 & 89030202 & 93011313 & 95080707 & 98100707 & 01092323 & 03120808 & 06062727 \\
\hline 85101111 & 89032727 & 93012525 & 95081313 & 98101919 & 01110505 & 03122020 & \\
\hline 85110202 & 89042525 & 93013131 & 95090505 & 98102727 & 01120202 & 03122727 & \\
\hline 85110909 & 89052323 & 93020707 & 95091010 & 98110505 & 01121515 & 03123131 & \\
\hline 85111313 & 89060606 & 93022020 & 95100202 & 98111414 & 01122424 & 04021212 & \\
\hline 85112929 & 89070505 & 93022828 & 95101818 & 98112323 & 02010707 & 04022727 & \\
\hline 85121010 & 89081414 & 93030808 & 95103030 & 98122525 & 02011919 & 04030909 & \\
\hline 85122828 & 89082727 & 93032020 & 95110505 & 99010606 & 02020505 & 04032525 & \\
\hline 86010606 & 89100808 & 93041212 & 95112727 & 99011313 & 02021111 & 04040505 & \\
\hline
\end{tabular}



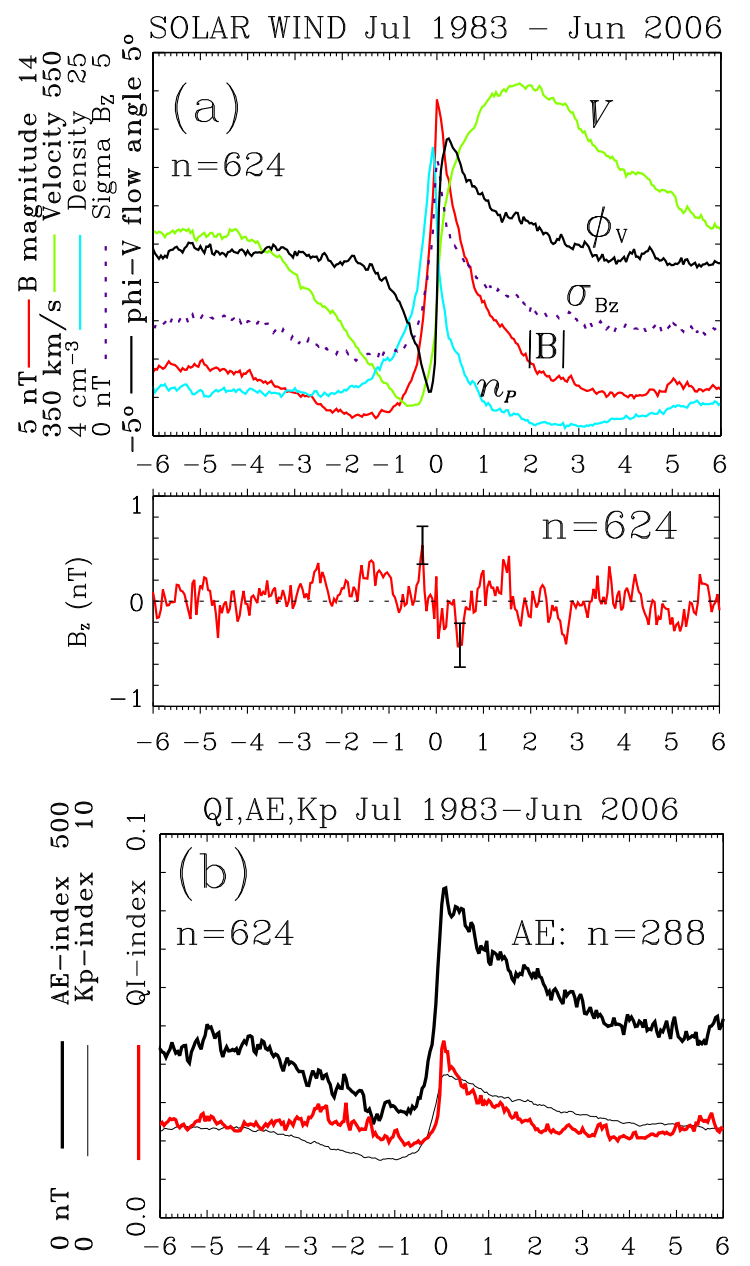
GREEN CORONA (530.3 nm, FeXIV)
July 1983 - December 2005 Solar wind $V_{M A X} \geqq 500 \mathrm{~km} / \mathrm{s}$

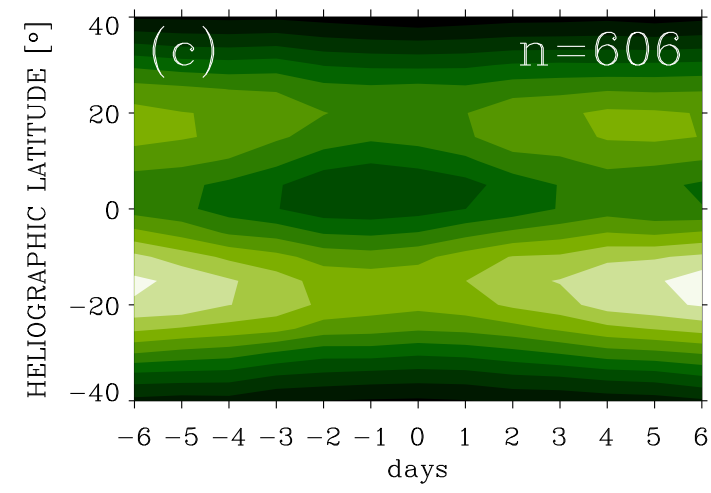

Fig. 10. Superposed epoch analysis of (a) solar wind plasma parameters $V, n_{p},|\mathbf{B}|, \sigma_{B z}, \phi_{V}$ and $B_{Z}$, (b) QI, AE and $K_{p}$ indices, and (c) the green coronal emission line intensity interpolated for the central meridian. Darker color represents lower intensity and contours are 2 ACUs apart. All time series are keyed by the arrival time of streams/CIRs. The AE-index was only available for years 1983-1988 and 1990-1995. calm interval before the arrival of high-speed streams. Two standard errors of the mean $\left( \pm 1 \sigma_{M}\right)$ before and after day 0 are shown.

As discussed in the companion paper (Prikryl et al., 2009), CIRs can be classified by IMF polarity following the method by Miyoshi and Kataoka (2008). For months around the spring and fall equinoxes, the mean IMF $B_{Z}$ component systematically shifts southward near the CIR interface for the group of events classified as type SBZ (southward $B_{Z}$ ), while for the group classified as NBZ (northward $B_{Z}$ ), the CIRs are associated with a northward shift in $B_{Z}$. In comparison with the method used by Miyoshi and Kataoka (2008), we have relaxed the selection criteria in order to allow most of the events used in the SPE analysis to be classified as SBZ or NBZ. We examined the IMF longitude (azimuthal) angle for $72 \mathrm{~h}$ after CIR (stream) interface to determine the direction of the IMF. The direction was defined to be away (+) from the Sun if more than $50 \%$ of the observed values were in the "away sector" (longitude angles between $45^{\circ}$ and $225^{\circ}$ ), or toward (-) the Sun if more than $50 \%$ of the observed values were in the "toward sector" (longitude angles between $225^{\circ}$ and $45^{\circ}$ ). Also, we extended the "spring" and "fall" equinox periods of February-May, and August-November used by Miyoshi and Kataoka (2008) to six months intervals of January-June and July-December approximately centered at equinoxes. Applying the so-called "spring-toward fallaway" rule, we classified CIRs into group A (type SBZ events) and B (type NBZ events). Because of the IMF data gaps the total number of events is less than the 624 CIRs used in Fig. 10. For each group, Fig. 11a and b shows the mean IMF $B_{Z}$ component for the Northern Hemisphere autumnwinter and spring-summer months, respectively. As expected, the SBZ events are more geo-effective than the NBZ events, which is shown for AE and $K_{p}$ indices in Fig. 11c-d. Other solar wind plasma parameters, including QI, show the same trends as those found in Fig. 10 for CIRs of type SBZ and NBZ (Miyoshi and Kataoka, 2008).

The CIR key times were also used in the SPE analysis of time series of the green corona intensity. Figure 10c shows the mean coronal hole centered on the sun disk on day -1 , or about 3 days before the maximum of the mean solar wind speed is observed near the Earth (Fig. 10a). The SPE analysis of the coronal intensity was repeated for two sub-periods before and after January 1995, as well as for 72 key times when geomagnetic indices were used as proxies indicating arrivals of CIRs. In all three cases (not shown) the results show a well defined coronal hole near the epoch day -1 , very similar to Fig. 10c thus validating the proxies that were used to identify these CIRs. 


\subsection{The SPE analysis of HCAI and statistical signifi- cance of the results}

Time series of HCAI spanning 23 years (July 1983 to June 2006) and constructed for $20^{\circ}$-wide latitude bands have the diurnal and seasonal/long-term variations of HCAI removed prior to the SPE analysis. As key times, 624 arrival times of CIRs at the leading edge of streams that reached maximum velocity $V_{\mathrm{MAX}} \geq 500 \mathrm{~km} / \mathrm{s}$ are used. Figure 12 shows the mean HCAI anomaly for 12 days centered at the key time (day 0) for the Northern (NH) and Southern (SH) Hemispheres. The mean variations over the 23-year interval are shown by black heavy lines. To check persistence or reproducibility of the mean HCAI variations we have initially split the data into two sub-periods 1983-1994 and 1995-2006. The most significant anomalies to be discussed below were found in both cases (not shown). To see if the detection of the effect on HCAI depends on the presence of volcanic aerosols, the data were subdivided into periods of high (1983-1986 and 1992-1996) and low (1987-1991 and 1997-2006) volcanic aerosol loading, for which the mean HCAI variations are shown by red dotted and green dashed lines, respectively. It is found that a decrease in HCAI to a minimum near day 0 , followed by an increase to a maximum a few days later, is a typical response to solar wind forcing. This temporal variation that is similar to the Wilcox effect for VAI is hereafter called the HCAI pattern or simply "pattern". The amplitude of the HCAI pattern, here defined as the extent of the negative to positive excursion, i.e. the difference between the overall mean HCAI anomaly maximum and minimum, is shown as a bold bar to the right of each curve. Each representative error bar that is shown for three points on each curve is \pm 1 standard error of the mean. The HCAI anomaly amplitude is considered significant at level $95 \%$ or above if it is equal or greater than $\pm 1.96 \sigma_{M}\left(3.92 \sigma_{M}\right)$, where $\sigma_{M}$ is the standard error of the mean averaged over 12 days. The observed amplitude in units of $\sigma_{M}$ is shown in Fig. 12.

Although standard errors of the mean indicate that much of the results discussed below have statistical significance at the 95\% level or above, this could be an overestimate (Shapiro, 1976; Hines and Halevy, 1977). Thus an additional test to assess the statistical significance using a randomization procedure is performed. We followed the approach adopted by Hines and Halevy (1977) and Williams (1978).

To estimate the statistical significance of the amplitude, the SPE analysis is done for $n$ randomly generated key times separated by at least 4 days and this is repeated for 100 ensembles of random key times. Thus, 100 values of the HCAI anomaly amplitudes (excursions) are computed for random key times. The mean amplitude and standard deviation $\sigma$ of the mean obtained from the SPE results for 100 sets of random key times and for each geographical sector are shown as two thin-line bars to the right of each plot. They represent the statistical noise level affecting the results of the SPE analysis. For comparison, the observed amplitude of the mean tempo-
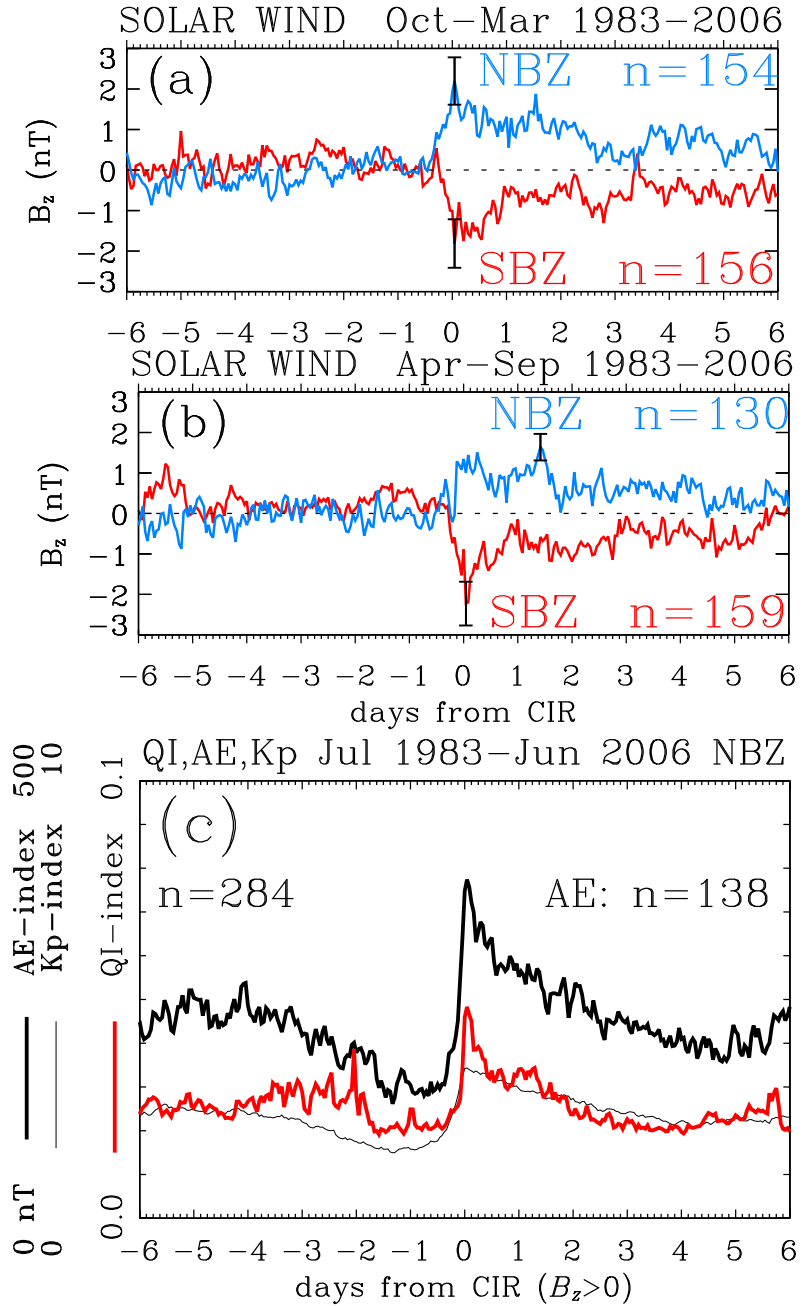

ㅇ- - QI,AE,Kp Jul 1983-Jun 2006 SBZ

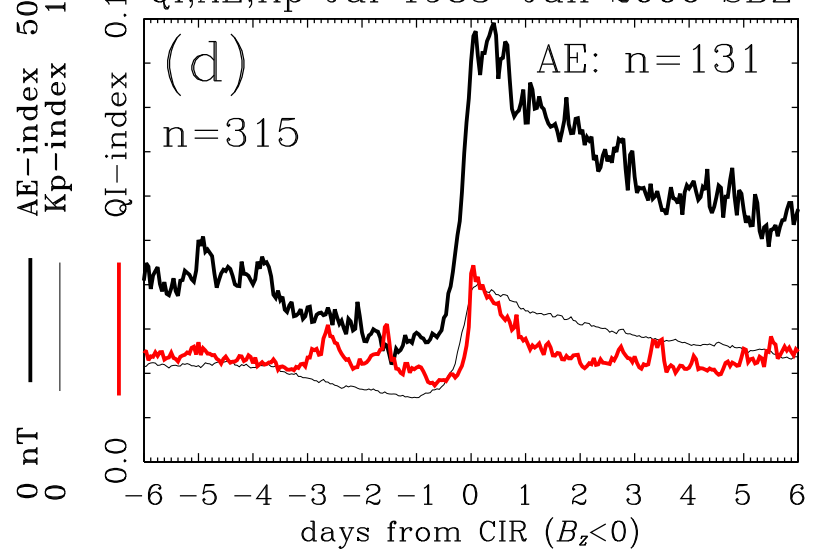

Fig. 11. Superposed epoch analysis of (a-b) the IMF $B_{Z}$ and (c-d) QI, AE and $K_{p}$ indices for NBZ and SBZ events.

ral variation for key times determined from the solar wind data is shown (bold bar). Based on this method of the significance estimation, if the observed mean amplitude exceeds the mean excursion for the randomly generated key times by 
HCAI anomaly 1983-2006

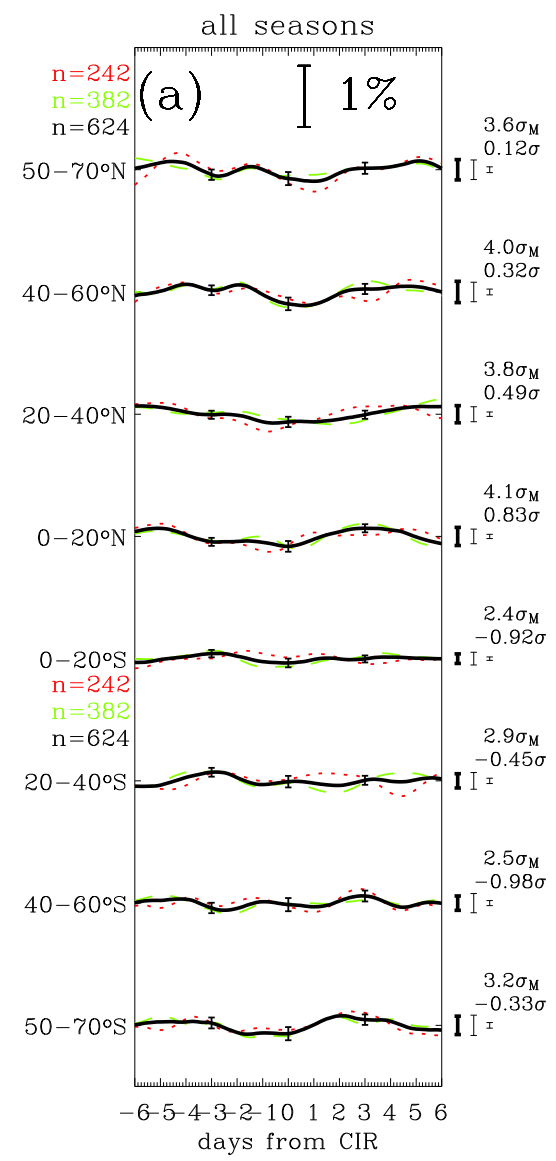

Longitude: $0-360^{\circ} \mathrm{E}$ Solar wind $V_{\text {MAx }} \geqq 500 \mathrm{~km} / \mathrm{s}$

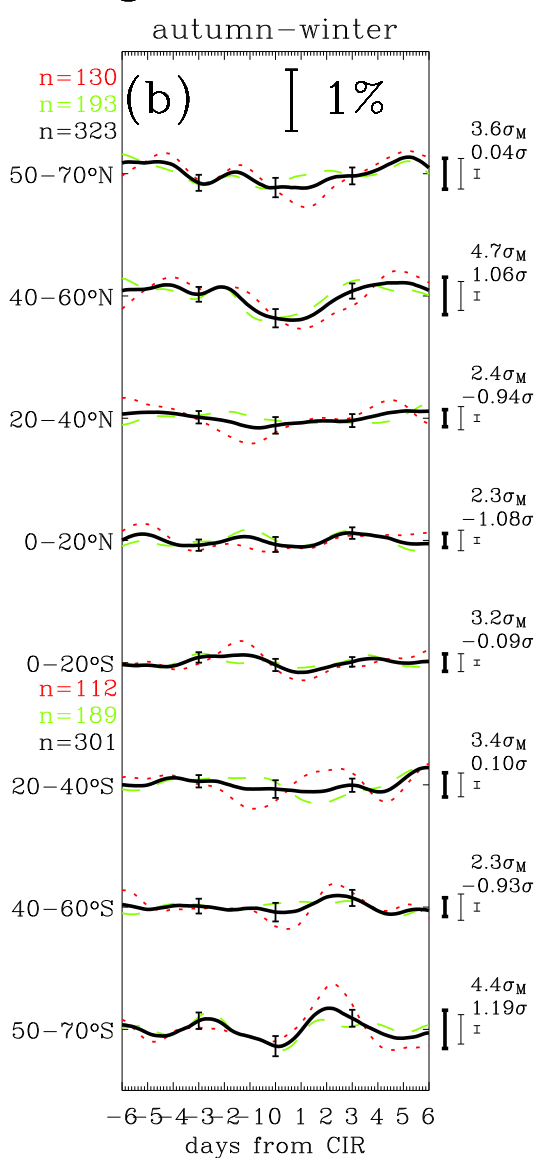

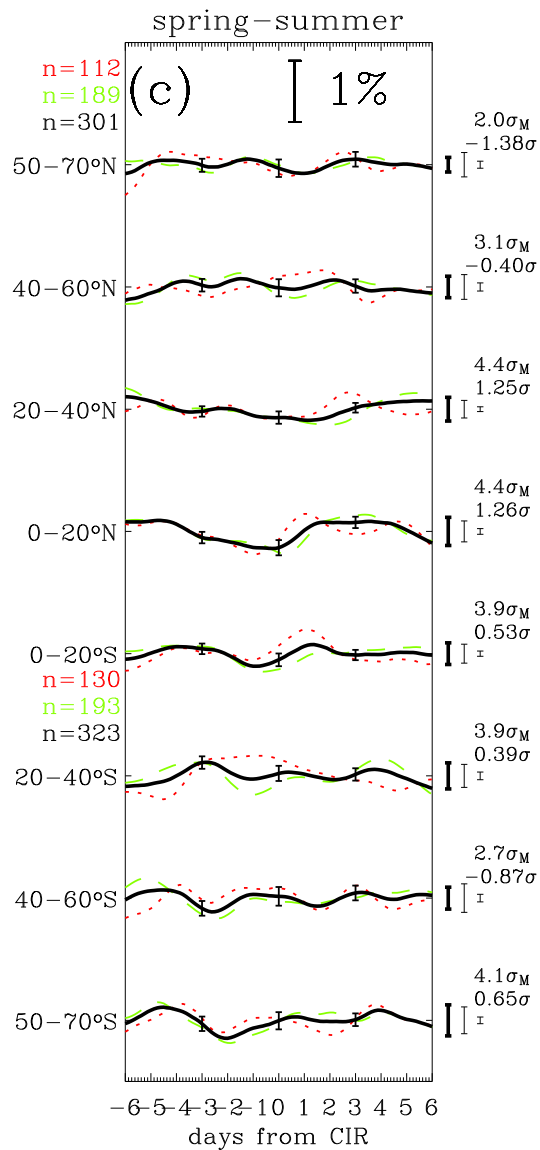

Fig. 12. The SPE analysis of high cloud area index (HCAI) for $20^{\circ}$-wide latitude bands for the Northern and Southern Hemispheres. The key times are the arrivals of solar wind streams with maximum velocity $V_{\mathrm{MAX}} \geq 500 \mathrm{~km} / \mathrm{s}$ for (a) all seasons, (b) autumn-winter and (c) springsummer. The HCAI anomaly for the whole time interval 1983-2006 (black line) and two sub-intervals consisting of years of high (dotted red line) and low (dashed green line) volcanic aerosol loading is shown. The bold bars on the right are the observed amplitudes over the 12-day period. The light bars are the mean amplitudes and standard deviation $\sigma$ of the mean for 100 sets of random key times representing the mean statistical noise. Departures $\Delta$ of the observed amplitude from the estimated statistical noise level in terms of $\sigma$ are shown. Representative error bars shown on each curve are two standard errors of the mean $\sigma_{M}$.

$1.96 \sigma$ or more the result is considered statistically significant at $95 \%$ level or above. The difference $\Delta$ between the observed maximum amplitude for the solar-wind CIR key times and the mean statistical-noise-related excursion is shown in units of $\sigma$ in Fig. 12.

It should be noted that the times of the HCAI anomaly excursions (maxima or minima) determined from analysis using 100 sets of random key times are randomly distributed over the 12-day period. This was checked by plotting minima and maxima distributions. In contrast, the observed temporal variations of HCAI show minima near day 0 and are followed by maxima a few days later. This lends an additional argument in support of the statistical significance of the results even if other estimates suggest less significant pattern amplitudes, particularly if such patterns are reproduced for two sub-intervals. Because of the observed persistence (re- producibility) of the pattern and its non-random phase, the significance test using 100 ensembles of randomly selected key times is underestimating the significance of the SPE results. Therefore, the significance level is likely between the value obtained by the randomization procedure and the possibly "overestimated" $\sigma_{M}$-based significance level as discussed above.

The all-season mean HCAI traces (Fig. 12a) show relatively small pattern amplitudes at mid-to-high $\left(40-60^{\circ} \mathrm{N}\right.$ and $\left.50-70^{\circ} \mathrm{S}\right)$ and low $\left(0-20^{\circ} \mathrm{N}\right)$ latitudes. The minimum near day 0 is followed by an increase to a maximum a few days later. These patterns persist through years of high and low aerosol loading but are better defined for the latter case because of the greater number of events. When the number of events in two subintervals is more balanced the patterns are better reproduced (not shown). The reproducibility and 
HCAI anomaly 1983-2006

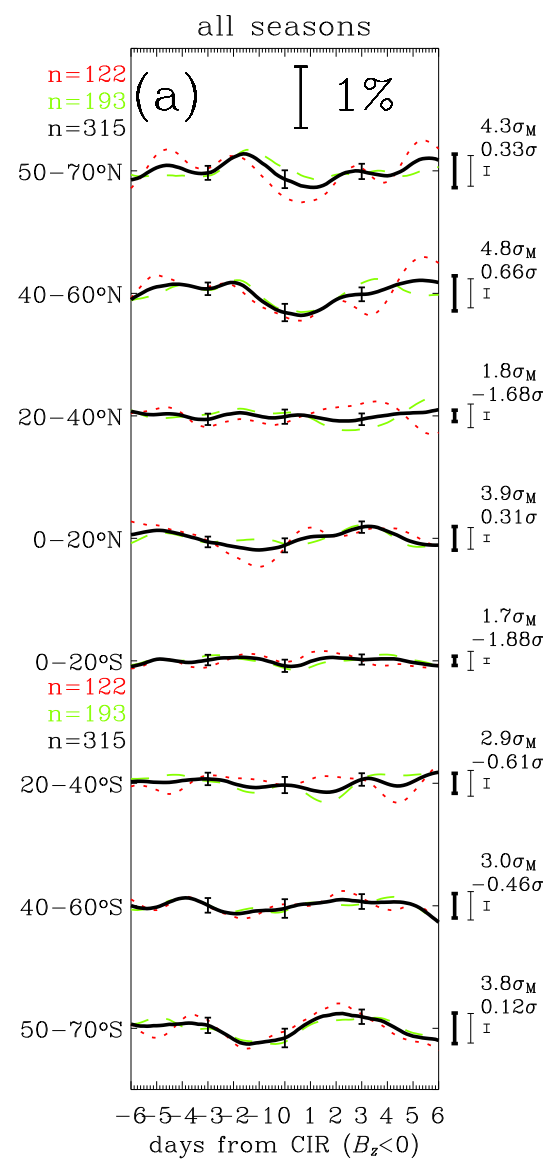

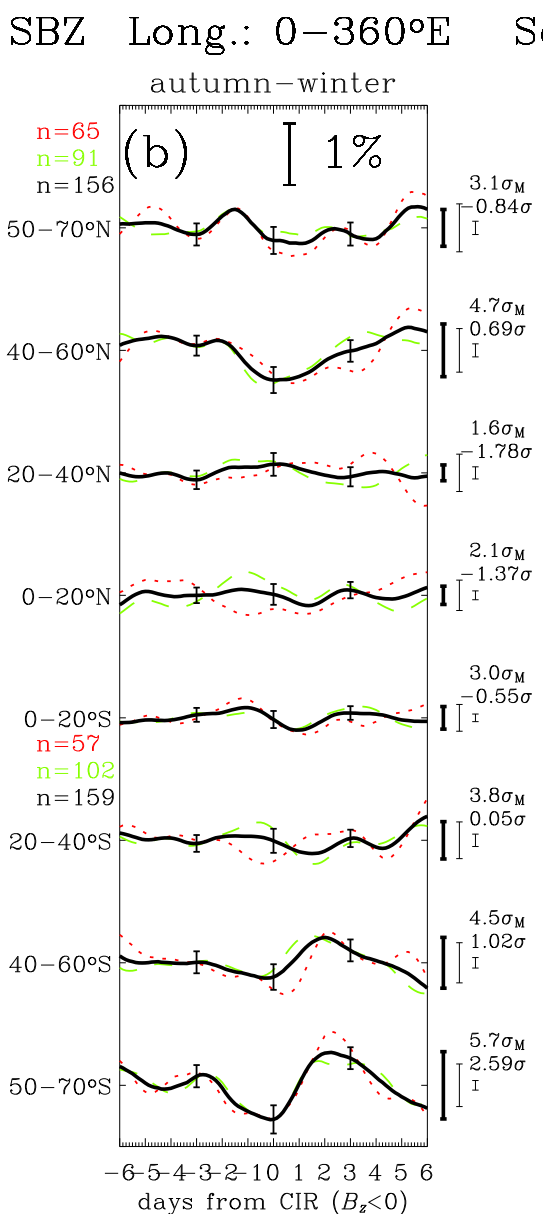

Solar wind $V_{M A X} \geqq 500 \mathrm{~km} / \mathrm{s}$

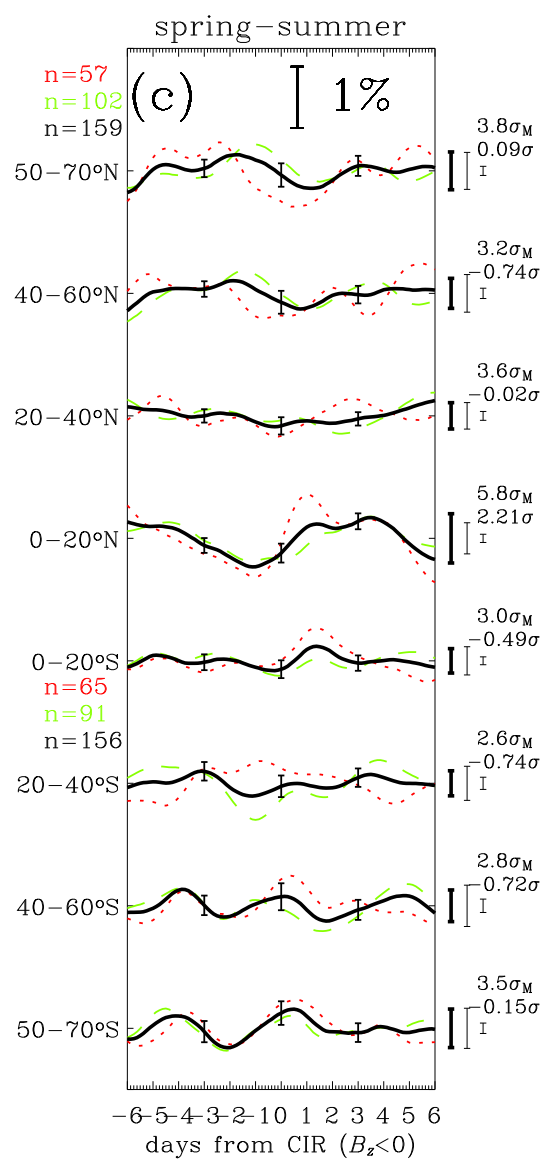

Fig. 13. The same as Fig. 12 but for SBZ events (IMF $\left.B_{Z}<0\right)$.

the fact that the amplitudes in some cases exceed $3.92 \sigma_{M}$ (latitude bins $40-60^{\circ} \mathrm{N}$ and $0-20^{\circ} \mathrm{N}$ ) suggest their statistical significance. Although small, the absolute amplitude of $\sim 0.3 \%$ represents a relative percentage fraction of about $1 \%$ of a typical average HCAI value of $30 \%$ at mid-to-high latitudes. When the amplitudes are compared with the mean statistical-noise-related excursion based on 100 ensembles of random key times, the difference $\Delta=0.83 \sigma$ or less. This test fails to support the statistical significance particularly at midto-high latitudes but, as discussed above, it underestimates the statistical significance.

Figure $12 \mathrm{~b}$ shows the SPE analysis results for the autumnwinter months (October-March and April-September for the $\mathrm{NH}$ and $\mathrm{SH}$, respectively). The amplitude of the HCAI pattern is doubled $(\sim 0.6 \%)$ and exceeds $\pm 2 \sigma_{M}\left(4 \sigma_{M}\right)$ and the patterns persist through years of high and low aerosol loading at mid-to-high latitudes. The difference $\Delta$ above the mean statistical noise level exceeds $1 \sigma$. The autumn-winter midlatitude HCAI anomaly and its statistical significance are very similar to Wilcox effect on vorticity area index (VAI). At low latitudes, the mean HCAI traces show only small excursions that are below the statistical noise level.
Figure $12 \mathrm{c}$ shows the SPE analysis results for the springsummer months (April-September and October-March for the $\mathrm{NH}$ and $\mathrm{SH}$, respectively). At mid-to-high latitudes, the traces flatten out or show more variability for the two sub-intervals. In contrast, the latitude bin $0-20^{\circ} \mathrm{N}$ shows a prominent and persistent pattern with amplitude above the noise level $(\Delta=1.26 \sigma)$. Judging from the closely reproduced amplitude of $\pm 2.1 \sigma_{M}$, the significance level is $96 \%$ or above. A similar pattern is also observed at southern latitudes $\left(0-20^{\circ} \mathrm{S}\right)$ but the overall amplitude is less significant.

The amplitudes of small but statistically significant HCAI responses to solar wind that we found at latitude bands 40 $70^{\circ}$ and $0-20^{\circ}$ in autumn-winter and spring-summer, respectively, are almost doubled when the SPE analysis is restricted to SBZ events (Fig. 13). As already discussed in Fig. 12a, the effect is found both in mid-to-high and low latitudes when all seasons are included (Fig. 13a). However, the mid-to-high-latitude response clearly occurs mainly in autumn-winter with high statistical significance, especially in the Southern Hemisphere (Fig. 13b), while the low-latitude response appears to be limited to spring-summer, particularly in the Northern Hemisphere (Fig. 13c). On the other 


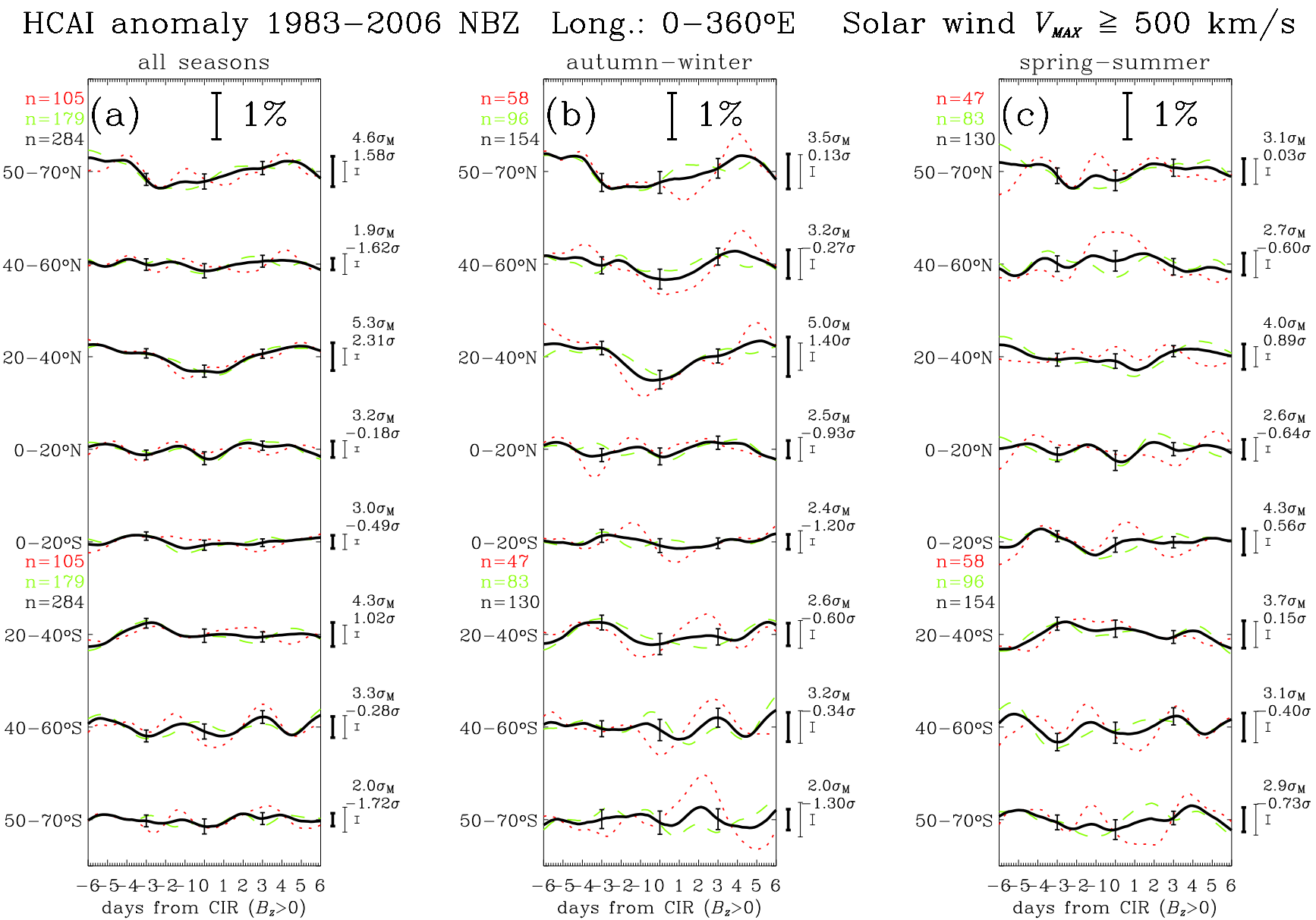

Fig. 14. The same as Fig. 12 but for NBZ events (IMF $B_{Z}>0$ ).

hand, for the NBZ events (Fig. 14), the autumn-winter effect weakens at latitudes $40-70^{\circ}$ but appears to be shifted to latitude band of $20-40^{\circ}$, particularly in the Northern Hemisphere where its statistical significance is high (Fig. 14a-b). No statistically significant and reproducible effect is found in spring-summer for the NBZ events (Fig. 14c).

In summary, for streams with $V_{\mathrm{MAX}} \geq 500 \mathrm{~km} / \mathrm{s}$, a statistically significant solar-wind-induced effect on the zonally stratified HCAI is found in autumn-winter and springsummer in the mid-to-high and low latitudes, respectively. The mean HCAI variation is very similar at these latitudes, namely a decrease to a minimum on the day of solar wind stream arrival followed by an increase to a maximum a few days later. The standard errors of the mean indicate statistical significance level exceeding $95 \%$. The estimates of statistical significance level are lower when the randomization procedure is used but this does not take into account the non-randomness of the phase of the observed effect and thus underestimates its statistical significance. The pattern of a decrease near the key day followed by an increase a few days later persists through years of high and low aerosol loading. Although the maximum absolute amplitudes of the mean temporal patterns (mid-latitude autumn-winter HCAI anomaly) is relatively small ( $\leq 0.6 \%$; in HCAI units) this represents a relative percentage fraction of up to about $2 \%$ for a typical average HCAI value of $30 \%$ at mid-to-high latitudes.

When the polarity of the IMF $B_{Z}$ is considered, these amplitudes are nearly doubled for events associated with the southward shift in $B_{Z}$. When the IMF $B_{Z}$ shifts northward at the CIR (stream) interface, the autumn-winter response at mid-to-high weakens or is shifted to intermediate latitudes and no statistically significant effect is found at low latitudes in spring-summer.

\section{Discussion}

We find, on the scale of days, a statistically significant response of the HCAI to high-speed solar wind from coronal holes. The mean HCAI decreases to a minimum near the arrival of high-speed plasma streams and then maximizes a few days later. This is a delayed response to a weakening solar wind that is being replaced by the next plasma stream. A statistically significant solar wind effect on highlevel cloudiness is found in the mid-to-high latitudes during 
autumn-winter and in low latitudes in spring-summer. The effect is significantly stronger for events associated with a southward shift of the IMF $B_{Z}$. Conversely, when the IMF $B_{Z}$ after the stream interface shifts northward, the autumnwinter effect weakens or shifts to lower (mid) latitudes while no statistically significant response is found at low latitudes in spring-summer. This is consistent with more intense auroral gravity waves being generated and propagated to low latitudes during geomagnetically disturbed periods, while the source of auroral gravity waves is at much higher latitudes when $B_{Z}$ is northward. The latter conditions may prevent the ducted waves from reaching the equator, although they may still reach mid latitudes.

The results presented in this paper, the verified and updated results on solar wind modulation of vorticity area index (Wilcox et al., 1974; Prikryl et al., 2009), and the observed tendency of significant extratropical storms to occur within a few days of arrival of high-speed plasma streams (Prikryl et al., 2009) all point to a link between the solar wind and the troposphere. The results indicate that interaction between solar wind generated auroral AGWs and weather systems is possible. As shown for the Wilcox effect on VAI (Prikryl et al., 2009), we found that the observed solar wind effect on HCAI persists through years of high and low volcanic aerosol and the mean amplitudes are similar for the northern and southern mid-to-high latitudes. The lack of dependence of the effect upon the presence of volcanic aerosols favours the AGW mechanism over the AEC mechanism, since the latter requires the presence of aerosols to reach a significant enough level to be detected. However, the AEC mechanism may still work provided there is less emphasis on the requirement of aerosols (Tinsley and Zhou, 2006).

It is important to understand the coupling process between auroral AGWs and weather systems. While there is a distinct possibility of amplification of the AGWs in that process, a more likely scenario involves the latent heat release which is a major source of energy of storms (Wernli et al., 2002). Latent heat release (or the reduction thereof) in extratropical cyclones is also invoked when discussing the possibility of electrically induced changes in contact ice nucleation by Tinsley et al. (1994). The VAI reduction has been attributed to a reduction in the scavenging of contact ice nuclei by supercooled droplets, and thus a reduction in the rate of glaciation of the winter storm clouds, leading to reductions in the precipitation efficiency and latent heat production. The AEC mechanism requires the presence of aerosols but the results here do not show a dependence on the volcanic aerosol presence. Furthermore, we have found that high-level clouds in tropical latitudes also show the same pattern of a decrease or negative anomaly in HCAI near the CIR arrival. This is not expected for the AEC mechanism, which relies on changes in the distribution of the vertical current density $J_{z}$ between high and low latitudes caused by conductivity variations in the stratosphere, due to relativistic electron flux variations. Tinsley and Zhou (2006, their Sects. 4.2 and 4.5) discuss lati- tude variations of $J_{z}$ with HCS crossing, namely the decrease in $J_{z}$ at high latitudes for a few days around HCS crossing as a result of reduced relativistic electron flux. Even in that case, the decrease in $J_{z}$ is conditioned by the presence of high concentration of volcanic aerosol. Clearly, the column resistance considerations would not explain a similar effect on clouds at tropical latitudes based on the same argument. Tinsley and Zhou (2006) concluded that "the short-term response to a HCS crossing would be a $J_{z}$ decrease at middle and high latitudes, accompanied by a smaller amplitude $J_{z}$ increase at low latitude". They note that this is the pattern observed for cloud responses to HCS crossings by Kniveton and Tinsley (2004, their Fig. 3f), namely that "cloud anomalies that were significant and negative were located in the Southern Hemisphere high and middle latitudes, and anomalies that were significant and positive were found in both hemispheres at low latitudes", for conditions of high aerosol loading and relativistic electron flux decrease. It is important to note that the latter result was shown for a very small statistical sample size of 21 HCS crossings. In the present study of solar wind influence on high-level clouds, the sample size is 624 CIRs. Regardless of aerosol loading, we find a decrease in HCAI at CIR crossing (corresponding to HCS crossing +1 day, on average) that is similar at mid-to-high and low latitudes, during autumn-winter and spring-summer periods, respectively.

We conclude that the present results are not consistent with the AEC model and favour the AGW mechanism. The latter does not depend on aerosol concentration and it should produce the same polarity of cloud anomalies at mid-to-high and low latitudes. If the solar wind generated auroral AGWs influence mesoscale processes and cloud band formation, this should be reflected in the cloud statistics as shown for HCAI.

If auroral AGWs can initiate slantwise convection by releasing the MSI, as suggested for Cases 1 and 2, latent heat release could result in rapid development and intensification of extratropical cyclones (Pauley and Smith, 1988; Kuo and Low-Nam, 1990). Even small-amplitude vertical motions due to auroral AGWs over a fraction of the wave period may lead to significant vertical lift of an air parcel to initiate the slantwise convection at near threshold MSI conditions. The cumulative effect of auroral AGWs could contribute sufficient latent heat release to power a storm. The case studies presented in this paper serve only as an illustration of a possible interaction of AGWs with extratropical cyclones. One of the limitations of the case studies of mesoscale bands is the small number of cases that have been examined in search of evidence of correlation with auroral AGWs. A network of instruments (e.g. radiometers) that can render time series of atmospheric parameters from which passing cloud bands can be detected and then correlated with auroral phenomena may help to identify events similar to Case 1 and 2. However, such observations of AGWs/TIDs that can be directly associated with sources in the auroral ionosphere are rare. This is partly due to weak radar signals occurring because HF propagation is disrupted when the ionosphere is highly structured. 
The resulting echoes are patchy and often there is neither sufficient ionospheric scatter in the AGW source region nor clarity in the TID signatures of the radar ground scatter. When potential sources of AGWs are observed, because of the dynamic nature of the solar wind coupling process, the AGW/TID source region is rarely fixed in latitude/longitude. This makes a one-to-one association with cloud bands difficult or impossible. Also, sources of AGWs both in the lower and upper atmosphere have been notoriously difficult to identify because of uncertainties of AGW propagation in a highly structured and dynamic atmosphere. The impact of neutral winds on auroral AGWs (Fig. 1e) is likely to be the most important factor that affects their propagation and thus their ability to influence tropospheric weather. However, this cannot be assessed without detailed knowledge of the spatial and temporal structure of the wind field.

Large scale AGWs/TIDs generated by auroral surges have been modelled (Balthazor and Moffett, 1997) and observed (Hajkovicz, 1991) to propagate as far as the equator. Medium-scale gravity waves can be ducted between the earth's surface and the lower thermosphere, can propagate undamped through the lower atmosphere over long distances (Hocke and Schlegel, 1996) and can leak from the lower atmosphere back to the thermosphere (Mayr et al., 1984a). Djuth et al. (2004) suggest that, near Arecibo, there is a continuum of gravity waves in the thermosphere, and that these showed "sets" of waves separated typically by $20-60 \mathrm{~min}$. Livneh et al. (2007) used incoherent scatter radar at Arecibo Observatory to observe quasi-coherent continuous waves with periods of $\sim 1 \mathrm{~h}$ in the ionosphere. These are also typical periods of auroral AGWs (Bristow and Greenwald, 1997) that are likely generated by pulsed ionospheric convection (Prikryl et al., 2005) virtually continuously and sometimes observed as TIDs by SuperDARN (Bristow et al., 1996; He et al., 2004). If auroral medium scale AGWs are observed in the ionosphere above Arecibo they must be ducted through the lower atmosphere waveguide and leak back to thermosphere; otherwise they would be damped and dissipate. The phase velocities of mediumscale gravity waves can be significantly lower than those of large-scale gravity waves and comparable to wind velocities (Fig. 1), thereby favoring the interaction of the wind and the waves.

If the auroral AGWs can release instabilities in the midlatitude cyclones as discussed in Sects. 5-6, and if the AGWs reach low latitudes because of ducted propagation in the wave guide between the surface/troposphere and the bottom thermosphere, they may exert similar influence on weather systems at low latitudes, including tropical cyclones. Ducted auroral AGWs may stimulate convection leading to mesoscale convective systems from which tropical cyclones develop. In mature tropical cyclones, high winds and large wind shears provide a favorable environment for the interaction between AGWs and the wind, with energy being transferred from the wave to the wind or vice versa. This may lead to strong amplification of the AGWs (McKenzie, 1972). The incident auroral AGWs would over-reflect in the cyclone sector where the wind is directed oppositely to the wave phase velocity (Cowling et al., 1971).

Similar to cloud/rain bands in extratropical cyclones such as Cases 1 and 2, spiral rain bands in developed tropical cyclones form sequentially and are wrapped around by cyclonic wind. In the cyclone sector where the incident auroral gravity wave phase velocity has the same direction as the wind, the wave would deposit its momentum, being absorbed if the horizontal phase velocity matches that of the wind (Booker and Bretherton, 1967). Assuming that the amplitudes of the auroral AGWs at tropospheric heights are small, this momentum would seem insignificant but a cumulative effect may not be negligible. Also, amplification of the AGWs is quite possible and could change their role in cloud formation dramatically. Similar to frontal rainbands and cloud heads of extratropical cyclones, the auroral AGWs incident on a tropical cyclone may modulate the slantwise convection, play a role in the excitation of spiral gravity waves by vortex Rossby waves (Schecter and Montgomery, 2004) and thus influence the tropical cyclone structure and intensity changes, which are not well understood (Wang and Wu, 2003).

A better understanding of the physical link between solar wind disturbances and extratropical cyclones may help in the forecasting of severe weather through a joint effort by the space and tropospheric weather research communities. Forecasting and modeling of space weather, particularly of the major CIR and CME arrivals, are now becoming possible, although the prediction of CME effects on the magnetosphere is still at an early stage (Maynard, 2000; McPherron and Siscoe, 2004; Schwenn et al., 2005; McPherron and Weygand, 2006). Tropospheric weather analysis and forecasting models have been developed for decades and have reached maturity (Lynch, 2002). Extratropical storms are known to develop from a strongly baroclinic environment. If such conditions are observed prior to an anticipated arrival of a CIR or CME and the intervening atmospheric conditions are favorable for auroral AGW propagation to reach the troposphere, further risk assessment of severe weather may be possible.

\section{Conclusions}

Observations of mesoscale cloud bands in extratropical cyclones following the arrival of high-speed plasma streams suggest a link between the solar wind and the mid-latitude troposphere, that link being atmospheric gravity waves. The gravity waves are generated by high-latitude ionospheric convection modulated by solar wind magneto-hydrodynamic waves coupling to the magnetosphere. The gravity waves then propagate in the upper and lower atmosphere. In the ionosphere they cause traveling ionospheric disturbances (TIDs) that are observed by HF radars. It is suggested that aurorally-forced atmospheric gravity waves release 
instabilities in the troposphere, thereby contributing to cloud formation in extratropical cyclones. In particular, the gravity waves may provide the lift required to release the moist symmetric instability, thus initiating slantwise convection and forming cloud/precipitation bands.

The circumstantial evidence of solar wind influence on clouds through auroral gravity waves as a link is supported by a statistical study of high-level cloudiness. A statistically significant response of the high-level cloud area index to fast solar wind from coronal holes is found in extratropical latitudes during autumn-winter and in tropical latitudes during spring-summer. The mean high-level cloud area index decreases to a minimum near the arrival of an interaction region at the leading edge of a high-speed plasma stream and then maximizes a few days later. In the extratropics, this response is consistent with the effect on tropospheric vorticity found by Wilcox et al. $(1973,1974)$ and verified by Prikryl et al. (2009). In the tropics, the observed highlevel cloud response is similar to that in mid-to-high latitudes, namely a decrease in high-level cloud cover at CIR arrival followed by an increase a few days later, which may be due to ducted auroral gravity waves stimulating convection in tropical storms and cyclones. The amplitudes of the responses at both the mid-to-high and low latitudes are almost doubled for high-speed plasma stream interfaces associated with a southward shift of the interplanetary magnetic field $B_{Z}$ component (SBZ events). This is consistent with more intense auroral gravity waves being generated and propagated to low latitudes during geomagnetically disturbed periods. For NBZ events, when the IMF $B_{Z}$ shifts northward after the passage of a stream interface past the Earth, the autumn-winter effect at mid-to-high latitudes weakens or shifts to lower (mid) latitudes and no statistically significant response is found at low latitudes in spring-summer. The source of auroral gravity waves is at much higher latitudes when $B_{Z}$ is northward, which may prevent the ducted waves from reaching the equator, although they may still reach the mid latitudes. For both the low and mid-to-high latitudes, the high-level-cloud response persists through years of low and high volcanic aerosol loading. In contrast, the AEC mechanism (Tinsley et al., 1994) requires the presence of stratospheric aerosols for a significant effect and, when the Earth is crossing the heliospheric current sheet, it is expected to produce negative and positive cloud anomalies in mid-to-high and low latitudes, respectively. However, if the requirement of aerosols for the AEC mechanism can be relaxed, the AGW and AEC mechanisms should work in synergy at least in midto-high latitudes.

Acknowledgements. We are grateful to many agencies, institutions and their projects, including CANOPUS (now CGSM), CIMSS, CSA, DMI, EUMETSAT, JHU/APL, MACCS, MSC, NASA, NOAA, NRCan, NSSDC and WDC, for services and data products on the Internet. We acknowledge contributions by the ACE, IMP8, SoHO, Wind, and SuperDARN teams. The ISCCP-D1 dataset was obtained from NASA Langley ASDC. The Prince George and
Saskatoon radar operations are funded by NSERC MFA Grants and CSA contracts, while the operations at Goose Bay and Kapuskasing have been funded by NASA and NSF grants. Much of the SuperDARN data analysis software and real-time data displays have been developed by R. Barnes, APL. The green corona dataset was provided by the Astronomical Institute of Slovak Academy of Sciences. Daily operation of the CRCC radiometer and ACTS beacon signal receivers was supported by Neville Reed. One of the authors (PP) is grateful to D. M. Schultz at NOAA for critical comments and suggestions, P. W. S. King at MSC for suggestions and for providing high-resolution cloud images, and to V. Pastirčák, Slovak Hydrometeorological Institute, for stimulating discussions.

Topical Editor M. Pinnock thanks one anonymous referee for her/his help in evaluating this paper.

\section{References}

Alfvén, H.: Existence of electromagnetic-hydrodynamic waves, Nature, 150, 405-406, 1942.

Amaya, C. A. and Rogers, D. V.: Propagation measurements campaign at two sites in Canada with the Advanced Communications technology Satellite, Proc. Sixth Ka Band Utilization Conf., Cleveland, 345-352, 2000.

Amaya, C. A. and Rogers, D. V.: Characteristics of rain fading on Ka-band satellite-earth links in a Pacific maritime climate, IEEE Trans. Microwave Theory and Techniques, 50(1), 41-45, 2002.

Balthazor, R. L. and Moffett, R. J.: A study of atmospheric gravity waves and travelling ionospheric disturbances at equatorial latitudes, Ann. Geophys., 15, 1048-1056, 1997, http://www.ann-geophys.net/15/1048/1997/.

Belcher, J. W. and Davis Jr., L.: Large-amplitude Alfvén waves in the interplanetary medium, 2, J. Geophys. Res., 76, 3534-3563, 1971.

Bennetts, D. A. and Hoskins, B. J.: Conditional symmetric instability - A possible explanation for frontal rainbands, Q. J. Roy. Meteorol. Soc., 105, 945-962, 1979.

Bertin, F., Testud, J., and Kersley, L.: Medium-scale gravity waves in the ionospheric F-region and their possible origin in weather disturbances, Planet. Space Sci., 23, 493-507, 1975.

Bjerknes, J.: On the structure of moving cyclones, Geofys. Publ., 1(2), 1-8, 1919.

Bjerknes, J. and Solberg, H.: Life cycles of cyclones and the polar front theory of atmospheric circulation, Geofys. Publ., 3(81), 3$18,1922$.

Bjerknes, V.: Über einen hydrodynamischen Fundamentalsatz und seine Anwendung besonders auf die Mechanik der Atmosphäre und des Weltmeeres, Konl. Svenska Vet.-Akad. Handl., 1898.

Bjerknes, V.: On the structure of the atmosphere when rain is falling, Q. J. Roy. Meteorol. Soc., 46, 119-140, 1920.

Bluestein, H. B.: Synoptic-Dynamic Meteorology in Midlatitudes, Volume II, in: Observations and Theory of Weather systems, Oxford University Press, p. 339-350, 1993.

Booker, J. R. and Bretherton, F. P.: The critical layer for internal gravity waves in a shear flow, J. Fluid Mech., 27, 513-539, 1967.

Borovsky, J. E. and Steinberg, J. T.: The "calm before the storm" in CIR/magnetosphere interactions: Occurrence statistics, solar wind statistics, and magnetospheric preconditioning, J. Geophys. Res., 111, A07S10, doi:10.1029/2005JA011397, 2006. 
Brekke, A.: On the relative importance of Joule heating and the Lorentz force in generating atmospheric gravity waves and infrasound waves in the auroral electrojets, J. Atmos. Terr. Phys., 41, 475-479, 1979.

Bristow, W. A. and Greenwald, R. A.: Multiradar observations of medium-scale acoustic gravity waves using the Super Dual Auroral Radar Network, J. Geophys. Res., 101, 24 499-24 511, 1996.

Bristow, W. A. and Greenwald, R. A.: On the spectrum of thermospheric gravity waves observed by the Super Dual Auroral Radar Network, J. Geophys. Res., 102(A6), 11 585-11 596, 1997.

Bristow, W. A., Greenwald, R. A., and Samson, J. C.: Identification of high-latitude acoustic gravity wave sources using the Goose Bay HF radar, J. Geophys. Res., 99, 319-331, 1994.

Bristow, W. A., Greenwald, R. A., and Villain, J. P.: On the seasonal dependence of medium-scale atmospheric gravity waves in the upper atmosphere at high latitudes, J. Geophys. Res., 101, 15 685-15 699, 1996.

Burns, G. B., Tinsley, B. A., Troshichev, O. A., FrankKamenetsky, A. V., and Bering, E. A.: Interplanetary magnetic field and atmospheric electric circuit influences on ground level pressure at Vostok, J. Geophys. Res., 112, D04103, doi:10.1029/2006JD007246, 2007.

Carslaw, K. S., Harrison, R. G., and Kirkby, J.: Cosmic rays, clouds and climate, Science, 298, 1732-1737, 2002

Chimonas, G. and Hines, C. O.: Atmospheric gravity waves launched by auroral currents, Planet. Space Sci., 18, 565-582, 1970.

Chimonas, G. and Hines, C. O.: Doppler ducting of atmospheric gravity waves, J. Geophys. Res., 91, 1219-1230, 1986.

Chisham, G., Lester, M., Milan, S. E., Freeman, M. P., Bristow, W. A., Grocott, A., McWilliams, K. A., Ruohoniemi, J. M., Yeoman, T. K., Dyson, P. L., Greenwald, R. A., Kikuchi, T., Pinnock, M., Rash, J. P. S., Sato, N., Sofko, G. J., Villain J.-P., and Walker, A. D. M.: A decade of the Super Dual Auroral Radar Network (SuperDARN): scientific achievements, new techniques and future directions, Surv. Geophys, 28, 33-109, doi:10.1007/s10712007-9017-8, 2007.

Cole, K. D.: Possible effects of solar variability on the middle atmosphere, J. Atmos. Terr. Phys., 46, 721-725, 1984.

Cowling, D. H., Webb, H. D., and Yeh, K. C.: Group rays of internal gravity waves in a wind stratified atmosphere, J. Geophys. Res., 79, 213-220, 1971.

Djuth, F. T., Sulzer, M. P., Gonzáles, S. A., Mathews, J. D., Elder, J. H., and Walterscheid, R. L.: A continuum of gravity waves in the Arecibo thermosphere?, Geophys. Res. Lett., 31, L16801, doi:10.1029/2003GL019376, 2004.

Doswell III, C. A., Brooks, H. E., and Maddox, R. A.: Flash flood forecasting: An ingredients-based methodology, Wea. Forecasting, 11, 560-581, 1996.

Emanuel, K. A.: Atmospheric Convection, Oxford University Press, 1994

Francis, S. H.: A theory of medium-scale traveling ionospheric disturbances, J. Geophys. Res., 79, 5245-5260, 1974.

Fritts, C. and Alexander, M. J.: Gravity wave dynamics and effects in the middle atmosphere, Rev. Geophys., 41(1), 1003, doi:10.1029/2001RG000106, 2003.

Fukao, S., Larsen, M. F., Yamanaka, M. D., Furukawa, H., Tsuda, T., and Kato, S.: Observations of a reversal in long-term average vertical velocities near the jet stream wind maximum, Mon.
Weather Rev., 119(6), 1479-1489, 1991.

Gosling, J. T., Asbridge, J. R., Bame, S. J., and Feldman, W. C.: Solar wind stream interfaces, J. Geophys. Res., 83, 1401-1412, 1978.

Greenwald, R. A., Baker, K. B., Dudeney, J. R., Pinnock, M., Jones, T. B., Thomas, E. C., Villain, J.-P., Cerrisier, J.-C., Senior, C., Hanuise, C., Hunsucker, R. D., Sofko, G., Koehler, J., Nielsen, E., Pellinen, R., Walker, A. D. M., Sato, N., and Yamagishi, H.: DARN/SUPERDARN A global view of the dynamics of highlatitude convection, Space Sci. Rev., 71, 761-796, 1995.

Hahn, C. J., Rossow, W. B., and Warren, S. G.: ISCCP Cloud properties associated with standard cloud types identified in individual surface observations, J. Climate, 14, 11-28, 2001.

Hajkowicz, L. A.: Auroral electrojet effect on the global occurrence pattern of large-scale traveling ionospheric disturbances, Planet. Space Sci., 39, 1189-1196, 1991.

Harrison, R. G. and Carslaw, K. S.: Ion-aerosol-cloud processes in the lower atmosphere, Rev. Geophys., 41(3), 1012 doi:10.1029/2002RG000114, 2003.

He, L.-S., Dyson, P. L., Parkinson, M. L., and Wan, W.: Studies of medium scale travelling ionospheric disturbances using TIGER SuperDARN radar sea echo observations, Ann. Geophys., 22, 4077-4088, 2004, http://www.ann-geophys.net/22/4077/2004/.

Hedin, A. E.: Extension of the MSIS thermosphere model into the middle and lower atmosphere, J. Geophys. Res., 96, 1159-1172, 1991.

Hernandez, G.: Vertical motions of the neutral thermosphere at mid latitude, Geophys. Res. Lett., 9, 555-557, 1982.

Hines, C. O.: Internal atmospheric gravity waves at ionospheric heights, Can. J. Phys., 38, 1441-1481, 1960.

Hines, C. O.: Motions of the neutral atmosphere, in: Physics of the Earth's upper atmosphere, edited by: Hines, C. O., Paghis, I., Hartz, T. R., and Fejer, J. A., Prentice-Hall, Inc., London, 1965.

Hines, C. O. and Halevy, I.: On the reality and nature of a certain sun-weather correlation, J. Atmos. Sci., 34, 382-404, 1977.

Hocke, K. and Schlegel, K.: A review of atmospheric gravity waves and travelling ionospheric disturbances: 1982-1995, Ann. Geophys., 14, 917-940, 1996, http://www.ann-geophys.net/14/917/1996/.

Houze Jr., R. A.: Cloud Dynamics, Academic Press, p. 53-56, 1993.

Hughes, W. J. and Engebretson, M. J.: MACCS: Magnetometer array for cusp and cleft studies, in the Satellite - Ground Based Coordination Sourcebook, ESA-SP-1198, edited by: Lockwood, M., Wild, M. N., and Opgenoorth, H. J., European Space Agency Publication, ESTEC, Noordwijk, The Netherlands, p. 119, 1997.

Hunsucker, R.: Estimate of the relative importance of Joule heating and the Lorentz force in generating atmospheric gravity waves from the auroral electrojet, J. Geophys. Res., 82(29), 4826-4828, 1977.

Hunsucker, R.: Atmospheric gravity waves generated in the highlatitude ionosphere: a review, Rev. Geophys. Space Phys., 20, 293-315, 1982.

Kirkland, M. W., Tinsley, B. A., and Hoeksema, J. T.: Are stratospheric aerosols the missing link between tropospheric vorticity and Earth transits of the heliospheric current sheet?, J. Geophys. Res., 101(D23), 29 689-29 699, 1996.

Kniveton, D. R. and Tinsley, B. A.: Daily changes in cloud cover and Earth transits of the heliospheric current sheet, J. Geophys. 
Res., 109, D11201, doi:10.1029/2003JD004232, 2004.

Koch, S. E. and O'Handley, C.: Operational forecasting and detection of mesoscale gravity waves, Wea. Forecasting, 12, 253-281, 1997.

Kuo, Y.-H. and Low-Nam, S.: Prediction of nine explosive cyclones over the western Atlantic ocean with a regional model, Mon. Weather Rev., 18, 3-25, 1990.

Lewis, R. V., Williams, P. J. S., Millward, G. H., and Quegan, S.: The generation and propagation of atmospheric gravity waves from activity in the auroral electrojet, J. Atmos. Terr. Phys., 58, 807-820, 1996.

Lin, Y., Lee, L. C., and Yan, M.: Generation of dynamic pressure pulses downstream of the bow shock by variations in the interplanetary magnetic field orientation, J. Geophys. Res., 101, 479493, 1996.

Lindblad, B. A.: Meteor radar rates, geomagnetic activity and solar wind sector structure, Nature, 273, 732-734, 1978.

Lindblad, B. A.: Solar control of meteor rates, in Proceedings of the ISCS 2003 Symposium: Solar Variability as an Input to the Earth's Environment, Tatranská Lomnica, ESA SP-535, 755759, 2003.

Lindzen, R. S. and Tung, K. K.: Banded convective activity and ducted gravity waves, Mon. Weather Rev., 104, 1602-1617, 1976.

Livneh, D. J., Seker, I., Djuth, F. T., and Mathews, J. D.: Continuous quasiperiodic thermospheric waves over Arecibo, J. Geophys. Res., 112(A7), A07313, doi:10.1029/2006JA012225, 2007.

Luhmann, J. G.: Atmospheric pressure and velocity fluctuations near the auroral electrojet, J. Geophys. Res., 85, 1749-1756, 1980.

Lundstedt, H.: Influence of interplanetary interaction regions on geomagnetic disturbances and tropospheric circulation, Planet. Space Sci., 32, 1541-1545, 1984.

Lynch, P.: Weather forecasting from wooly art to solid science, in: Meteorology at the Millennium, pp. 106-119, Academic Press, London, 2002.

Marsh, N. D. and Svensmark, H.: Low cloud properties influenced by cosmic rays, Phys. Rev. Lett., 85(23), 5004-5007, 2000.

Marsh, N. and Svensmark, H.: Comment on "Solar influences on cosmic rays and cloud formation: A reassessment" by Bomin Sun and Raymond S. Bradley, J. Geophys. Res., 109, D14205, doi:10.1029/2003JD004063, 2004.

Mastrantonio, G., Einaudi, F., and Fua, D.: Generation of gravity waves by jet streams in the atmosphere, J. Atmos. Sci., 33, 17301738, 1976.

Maynard, N.: Space weather prediction, in: Storms, vol. II, edited by: Pielke, R. and Pielke, R., Routledge, London, 2000.

Mayr, H. G., Harris, I., Varosi, F., and Herrero, F. A.: Global excitation of wave phenomena in a dissipative multiconstituent medium 1. Transfer function of the Earth's thermosphere, J. Geophys. Res., 89, 10 929-10959, 1984a.

Mayr, H. G., Harris, I., Varosi, F., and Herrero, F. A.: Global excitation of wave phenomena in a dissipative multiconstituent medium 2. Impulsive perturbations in the Earth's thermosphere, J. Geophys. Res., 89, 10 961-10 986, 1984b.

McKenzie, J. F.: Reflection and amplification of acoustic-gravity waves at a density and velocity discontinuity, J. Geophys. Res., 77, 2915-2926, 1972.

McPherron, R. L. and Siscoe, G.: Probabilistic forecasting of ge- omagnetic indices using solar wind air mass analysis, Space Weather, 2, S01001, doi:10.1029/2003SW000003, 2004.

McPherron, R. L. and Weygand, J.: The solar wind and geomagnetic activity as a function of time relative to corotating interaction regions, in: Recurrent Magnetic Storms: Corotating Solar Wind Streams, edited by: Tsurutani, B., McPherron, R. L., Gonzalez, W., Lu, G., Sobral, J. H. A., Gopalswamy, N., AGU Monograph, 167, 125-137, 2006.

Millward, G. H., Quegan, S., Moffett, R. J., Fuller-Rowell, T. J., and Rees, D.: A modeling study of the coupled ionospheric and thermospheric response to an enhanced high-latitude electric field event, Planet. Space Sci., 41, 45-56, 1993 a.

Millward, G. H., Moffett, R. J., Quegan, S., and Fuller-Rowell, T. J.: Effect of an atmospheric gravity wave on the midlatitude ionospheric F layer, J. Geophys. Res., 98, 19 173-19 179, 1993 b.

Millward, G. H.: A resonance effect in AGWs created by periodic recurrent bursts in the auroral electric field, Ann. Geophys., 12, 94-96, 1994, http://www.ann-geophys.net/12/94/1994/.

Miyoshi, Y. and Kataoka, R.: Flux enhancement of the outer radiation belt electrons after the arrival of stream interaction regions, J. Geophys. Res., 113, A03S09, doi:10.1029/2007JA012506, 2008.

Nastrom, G. D., Peterson, M. R., Green, J. L., Gage, K. S., and VanZandt T. E.: Sources of gravity wave activity seen in the vertical velocities observed by the Flatland VHF Radar, J. Appl. Meteorol., 29(8), 783-792, 1990.

Nastrom, G. D. and VanZandt, T. E.: Mean vertical motions seen by radar wind profilers, J. Appl. Meteorol., 33(8), 984-995, 1994.

Oliver, W. L., Otsuka, Y., Sato, M., Takami, T., and Fukao, S.: A climatology of $\mathrm{F}$ region gravity waves propagation over the middle and upper atmosphere radar, J. Geophys. Res., 102, 14449 $14512,1997$.

Osherovich, V. A., Fainberg, J., and Stone, R. G.: Solar wind quasiinvariant as a new index of solar activity, Geophys. Res. Lett., 26(16), 2597-2600, 1999.

Oyama, S., Watkins, B. J., Maeda, S., Shinagawa, H., Nozawa, S., Ogawa, Y., Brekke, A., Lathuillere, C., and Kofman W.: Generation of the lower-thermospheric vertical wind estimated with the EISCAT KST radar at high latitudes during periods of moderate geomagnetic disturbance, Ann. Geophys., 26, 1491-1505, 2008, http://www.ann-geophys.net/26/1491/2008/.

Øieroset M., Sandholt, P. E., Lühr, H., Denig, W. F., and Moretto, T.: Auroral and geomagnetic events at cusp/mantle latitudes in the prenoon sector during positive IMF $B_{y}$ conditions: Signatures of pulsed magnetopause reconnection, J. Geophys. Res., 102, 7191-7205, 1997.

Pallé, E., Goode, P. R., Montaés-Rodriguez, P., and Koonin, S. E.: Can earth's albedo and surface temperatures increase together?, Eos Transactions, American Geophysical Union, 87(4), 37-43, 2006.

Pauley, P. M. and Smith, P. J.: Direct and indirect effects of latent heat release on a synoptic-scale wave system, Mon. Weather Rev., 116(5), 1209-1236, 1988.

Pinnock, M., Rodger, A. S., Dudeney, J. R., Greenwald, R. A., Baker, K. B., and Ruohoniemi, J. M.: An ionospheric signature of possible enhanced magnetic field line merging on the dayside magnetopause, J. Atmos. Terr. Phys., 53, 201-212, 1991.

Prikryl, P.: Green corona, geomagnetic activity and radar meteor rates, Bull. Astron. Inst. Czechosl., 30, 321-330, 1979. 
Prikryl, P., Muldrew, D. B., and Sofko, G. J.: High-speed solar wind, auroral electrojets and atmospheric gravity waves: A link to the Earth's atmosphere, in Proceedings of the ISCS 2003 Symposium: Solar Variability as an Input to the Earth's Environment, Tatranska Lomnica, ESA SP-535, 371-376, 2003.

Prikryl, P., Muldrew, D. B., Sofko, G. J., and Ruohoniemi, J. M.: Solar wind Alfvén waves: A source of pulsed ionospheric convection and atmospheric gravity waves, Ann. Geophys., 23, 401417, 2005, http://www.ann-geophys.net/23/401/2005/.

Prikryl, P., Rušin, V., and Rybanský, M.: The influence of solar wind on extratropical cyclones - Part 1: Wilcox effect revisited, Ann. Geophys., 27, 1-30, 2009,

http://www.ann-geophys.net/27/1/2009/.

Provan, G., Yeoman, T. K., and Milan, S. E.: CUTLASS Finland radar observations of the ionospheric signatures of flux transfer events and the resulting plasma flows, Ann. Geophys., 16, 14111422, 1998, http://www.ann-geophys.net/16/1411/1998/.

Rees, D., Smith, R. W., Charleton, P. J., McCormac, F. G., Lloyd, N., and Steen, Å.: The generation of vertical thermospheric winds and gravity waves at auroral latitudes-I. Observations of vertical winds, Planet. Space Sci., 32(6), 667-684, 1984.

Roberts, W. O. and Olson, R. H.: Geomagnetic storms and wintertime 300-mb trough development in the North Pacific-North America Area, J. Atmos. Sci., 30, 135-140, 1973.

Roldugin, V. C. and Tinsley, B. A.: Atmospheric transmission changes associated with solar wind - induced ionization variations, J. Atmos. Solar Terr. Phys., 66, 1143-1149, 2004.

Rossow W. B. and Schiffer, R. A.: Advances in understanding clouds from ISCCP, B. Am. Meteorol. Soc., 80, 2261-2287, 1999.

Rostoker, G., Samson, J. C., Creutzberg, F., Hughes, T. J., McDiarmid, D. R., McNamara, A. G., Vallance Jones, A., Wallis, D. D., and Cogger, L. L.: CANOPUS - a ground-based instrument array for remote sensing the high-latitude ionosphere during the ISTP/GGS program, Space Sci. Rev., 71, 743-760, 1995.

Ruohoniemi, J. M. and Baker, K. B.: Large-scale imaging of highlatitude convection with Super Dual Auroral Radar Network HF radar observations, J. Geophys. Res., 103, 20 797-20 811, 1998.

Rybanský, M., Rušin, V., and Minarovijech, M.: Coronal index of solar activity, Space Sci. Rev., 95, 227-234, 2001.

Rybanský, M., Rušin, V., Minarovjech, M., Klocok, L., and Cliver, E. W.: Reexamination of the coronal index of solar activity, J. Geophys. Res., 110, A08106, doi:10.1029/2005JA011146, 2005.

Samson, J. C., Greenwald, R. A., Ruohoniemi, J. M., and Baker, K. B.: High-frequency radar observations of atmospheric gravity waves in the high latitude ionosphere, Geophys. Res. Lett., 16, 875-878, 1989.

Samson, J. C., Greenwald, R. A., Ruohoniemi, J. M., Frey, A., and Baker, K. B.: Goose Bay radar observations of earth-reflected atmospheric gravity waves in the high-latitude ionosphere, J. Geophys. Res., 95, 7693-7709, 1990.

Schecter, D. A. and Montgomery, M. T.: Damping and pumping of a vortex Rossby wave in a monotonic cyclone: Critical layer stirring versus inertia-buoyancy wave emission, Phys. Fluids, 16, 1334-1348, 2004.

Schultz, D. M. and Schumacher, P. N.: The use and misuse of conditional symmetric instability, Mon. Weather Rev., 127, 27092732, Corrigendum, 128, 1573, 1999.

Schwenn, R., Dal Lago, A., Huttunen, E., and Gonzalez, W. D.:
The association of coronal mass ejections with their effects near the Earth, Ann. Geophys., 23, 1033-1059, 2005,

http://www.ann-geophys.net/23/1033/2005/.

Shapiro, R.: Solar magnetic sector structure and terrestrial atmospheric vorticity, J. Atmos. Sci., 33, 865-870, 1976.

Siscoe, G. L.: Structure and orientation of solar wind interaction fronts, J. Geophys. Res., 77, 27-34, 1972.

Stauning, P., Friis-Christensen, E., Rasmussen, O., and Vennerstrøm, S.: Progressing polar convection disturbances: Signature of an open magnetosphere, J. Geophys. Res., 99, 11303-11317, 1994.

Stewart, R. E., Szeto, K. K., Reinking, R. F., Clough, S. A., and Ballard, S. P.: Midlatitude cyclonic cloud systems and their features affecting large scales and climate, Rev. Geophys., 36(2), 245-273, 1998.

Sun, B. and Bradley, R. S.: Solar influences on cosmic rays and cloud formation: A reassessment, J. Geophys. Res., 107(D14), 4211, doi:10.1029/2001JD000560, 2002.

Svensmark, H. and Friis-Christensen, E.: Variation of cosmic ray flux and global cloud coverage - a missing link in solar-climate relationships, J. Atmos. Solar-Terr. Phys., 59(11), 1225-1232, 1997.

Thorpe, A. J.: Extratropical cyclones An historic perspective, in: Meteorology at the Millennium, pp. 14-22, Academic Press, London, 2002.

Tinsley, B. A.: Influence of solar wind on the global electric circuit, and inferred effects on cloud microphysics, temperature, and dynamics in the troposphere, Space Sci. Rev., 94, 231-258, 2000.

Tinsley, B. A. and Deen, G.: Apparent tropospheric response to $\mathrm{MeV}-\mathrm{GeV}$ particle flux variations: a connection via electrofreezing of supercooled water in high level clouds?, J. Geophys. Res., 96, 22 283-22 296, 1991.

Tinsley, B. A. and Zhou, L.: Initial results of a global circuit model with stratospheric and tropospheric aerosols, J. Geophys. Res., 111, D16205, doi:10.1029/2005JD006988, 2006.

Tinsley, B. A., Rohrbaugh, R. P., and Hei, M.: Electroscavenging in clouds with broad droplet size distributions and weak electrification, Atmos. Res., 59-60, 115-136, 2001.

Tinsley, B. A., Zhou, L., and Plemmons, A.: Changes in scavenging of particles by droplets due to weak electrification in clouds, Atmos. Res., 79, 266-295, 2006.

Tinsley, B. A., Burns, G. B., and Zhou, L.: The role of the global circuit in solar and internal forcing of clouds and climate, Adv. Space Res., 40, 1126-1139, 2007.

Tinsley, B. A., Hoeksema, J. T., and Baker, D. N.: Stratospheric volcanic aerosols and changes in air-earth current density at solar wind magnetic sector boundaries as conditions for Wilcox tropospheric vorticity effect, J. Geophys. Res., 99(D8), 16 805$16813,1994$.

Todd, M. C. and Kniveton, D. R.: Changes in cloud cover associated with Forbush decreases of galactic rays, J. Geophys. Res., 106, 32 031-32 041, 2001.

Tsurutani, B. T. and Gonzalez, W. D.: The cause of high intensity long-duration continuous AE activity (HILDCAAs): Interplanetary Alfvén wave trains, Planet. Space Sci., 35, 405-412, 1987.

Uccellini, L. W.: A case study of apparent gravity wave initiation of severe convective storms, Mon. Weather Rev., 103, 497-513, 1975.

Vincent, R. A.: Gravity wave motions in the mesosphere, J. Atmos. 
Terr. Phys., 46, 119-128, 1984.

Waldock, J. A. and Jones, T. B.: Source regions of medium scale traveling ionospheric disturbances observed at mid-latitudes, J. Atmos. Terr. Phys., 49, 105-114, 1987.

Wang, Y. and Wu, C.-C.: Current understanding of tropical cyclone structure and intensity changes - A review, Meteor. Atmos. Phys., 87, 257-278, doi:10.1007/s00703-003-0055-6, 2004.

Wernli, H., Dirren, S., Liniger, M. A., and Zillig, M.: Dynamical aspects of the life cycle of the winter storm 'Lothar' (24-26 December 1999), Q. J. Roy. Meteorol. Soc., 128, 405-430, 2002.

Wilcox, J. M., Scherrer, P. H., Svalgaard, L., Roberts, W. O., and Olson, R. H.: Solar magnetic sector structure: Relation to circulation of the earth's atmosphere, Science, 180, 185-186, 1973.
Wilcox, J. M., Scherrer, P. H., Svalgaard, L., Roberts, W. O., Olson, R. H., and Jenne, R. L.: Influence of solar magnetic sector structure on terrestrial atmospheric vorticity, J. Atmos. Sci., 31, 581-588, 1974.

Wilcox, J. M., Svalgaard, L., and Scherrer, P. H.: On the reality of a sun-weather effect, J. Atmos. Sci., 255, 1113-1116, 1976.

Williams, R. G.: A study of the energetics of a particular sunweather relation, Geophys. Res. Lett., 5(6), 519-522, 1978.

Yeh, K. C. and Liu, C. H.: Acoustic-gravity waves in the upper atmosphere, Rev. Geophys. Space Phys., 12, 193-216, 1974.

Zhou, L. and Tinsley, B. A.: Production of space charge at the boundaries of layer clouds, J. Geophys. Res., 112, D11203, doi:10.1029/2006JD007998, 2007. 